\title{
Aspetti della dinamica di rocce, calcestruzzo ed acque
}

\author{
Pigtro Oalom
}

Ricevuto il 20 Marzo 1962

1. - In un lavoro precedente, destinato ad una Rivista tecnica $\left({ }^{22}\right)$, ho esposto le possibilità della geofisica (o, almeno, di parte della geofisiea) nella ricerea delle condizioni ambiente più alatte alla costruzione di una grande diga, nonehé - ma volta costruito lo sbarramento - nel fornire i mezzi a i metodi per un controllo efficace, sensibile e continuo della sua stabilità.

Desidero qui approfondire aleuni aspetti dei problemi che si presentano in queste indagini, sui quali nel lavoro eitato ho dovuto sorvolare.

Ho detto dell'utilità di un'indagine preliminare, condotta con clinografi, allo scopo di stabilire l'unicità del bloceo geodetico, destinato a sopportare una diga. IIo aceennato pure allopportunita di una ricereat dei periodi predominanti nella zona prescelta per l'ancoraggio della diga, allo scopo di evitare la costruzione del mamufatto su eulle roceiose animate da periodi propri, che rientrino tra quelli più frequenti nelle zone epicentrali. Fatto da evitare soplatutto se solo una parte della diga risultasse ancorata ad uno sperone con periodi liberi dell'ordine detto, creando pericolose dissimmetrie in "ampo dinamico. Ia sismologia ha già most lato quali sono i periodi che maggiomente predominano nelle zone epicentrali (v. fig. 1). Nelle indagini compiute in numerose valli alpine, ho aruto modo di riscontrare sovente oscillazioni libere trasversali ("flexural waves") in speroni di roceid, che averano resistito allerosione e che si sarebbero prestati ottimamente allancoraggio di una diga, arenti periodi di quell'ordine $(0,2$ sec. cirea) (v. fige. 2 e 3$)$. Talrolta, tali speroni ap)paiono mascherati da sovrastrutture. Specie in zone sogrette a movimenti sismici, sono rigorosamente da evitare situazioni del genere.

IIo già riferito a lungo sull'ausilio prezioso del vibrometro nel rilevamento delle caratteristiche elastiche del mezzo. Ho osservato come tali calatteristiche varino da luogo a luogo e, nello stesso posto, secondo 
le direzioni. Ho dimostrato ehe, nell'ambito delle elevate frequenze, esiste il fenomeno della dispersione anomala, nel senso che la velocità di gruppo riesse maggiore della velocita di fase delle onde componenti, per cui il fronte ì costitnito dalle onde associate ai più brevi periodi.
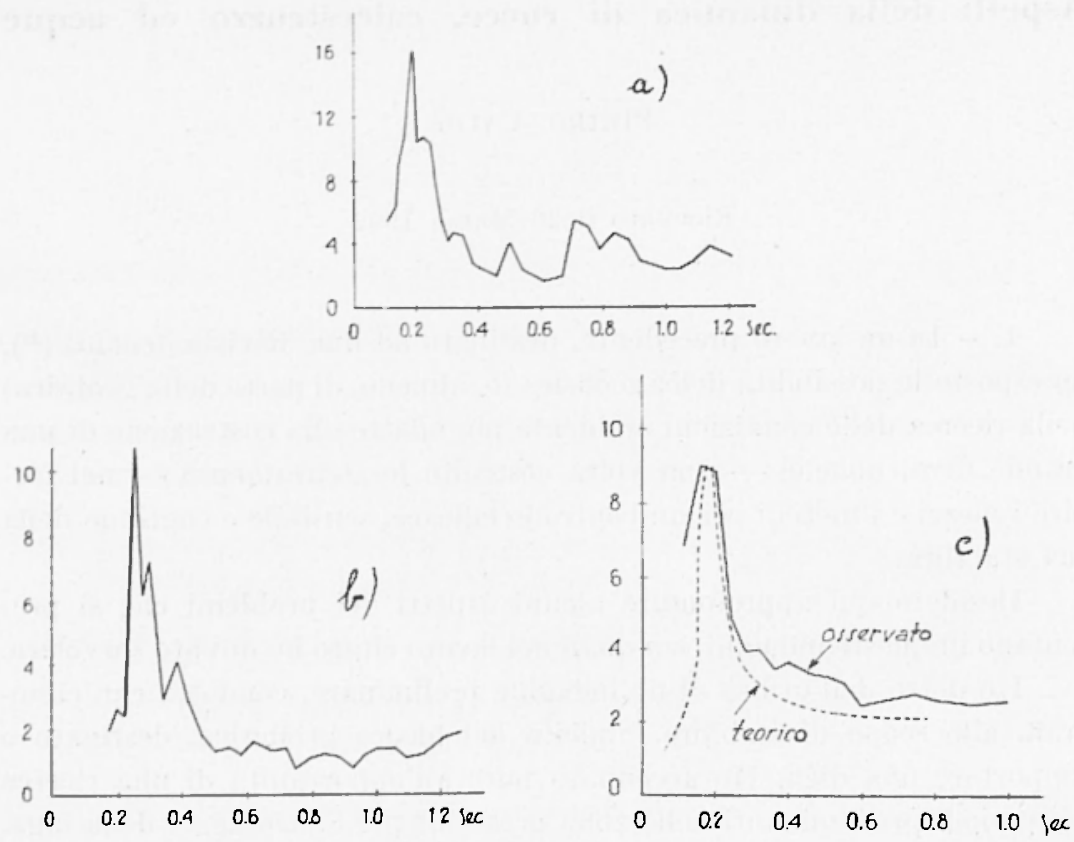

Fig. 1 - a) Rapporto ampiezze osservate in superficie o a $300 \mathrm{~m}$ di profondità nella miniera di Ilitachi (Giappone);

b) Rapporto ampiezze osservate in superficie e a $21 \mathrm{~m}$ di profondita a Tokai-mura (Giappone);

c) Rapporto medio ampiezze fra 0 e $300 \mathrm{~m}$ di profondita nella miniera di Ilitacla (Giappone).

Pertanto, più il mezzo presenta elevate le sue caratteristiche elastiche -.e quindi più alte le velocità delle onde longitudinali e trasversali - più alta risulta la frequenza d'onda. E questo un altro elemento qualitativo nella valutazione della compattezza di un mezzo solido. Ho ampiamente documentato altrove la realtà di questo fenomeno. Qui mi limito a riportare gli esempi delle figg. $4,5,6,7$. Più il mezzo è rilassato più lungo diviene il periodo dell'onda sismica che in esso insorge. In coltri alluvionali cio riesce di chiara evidenza (fig. 6). Qualora il fondo roccioso, sopportando un tratto di terreno coperto da materiale sciolto, sia a non grande 


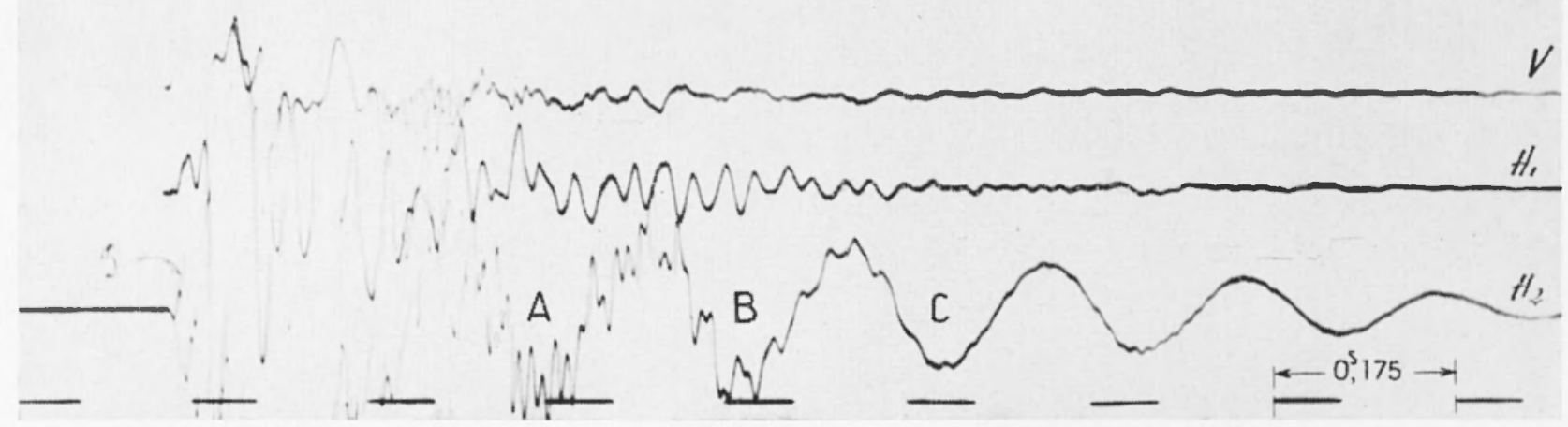

Fig, 2 - Oseillazioni con periodo di 0,2 sec. eirca (AB-BC....) osservate nella valle dell'Ambiesta.

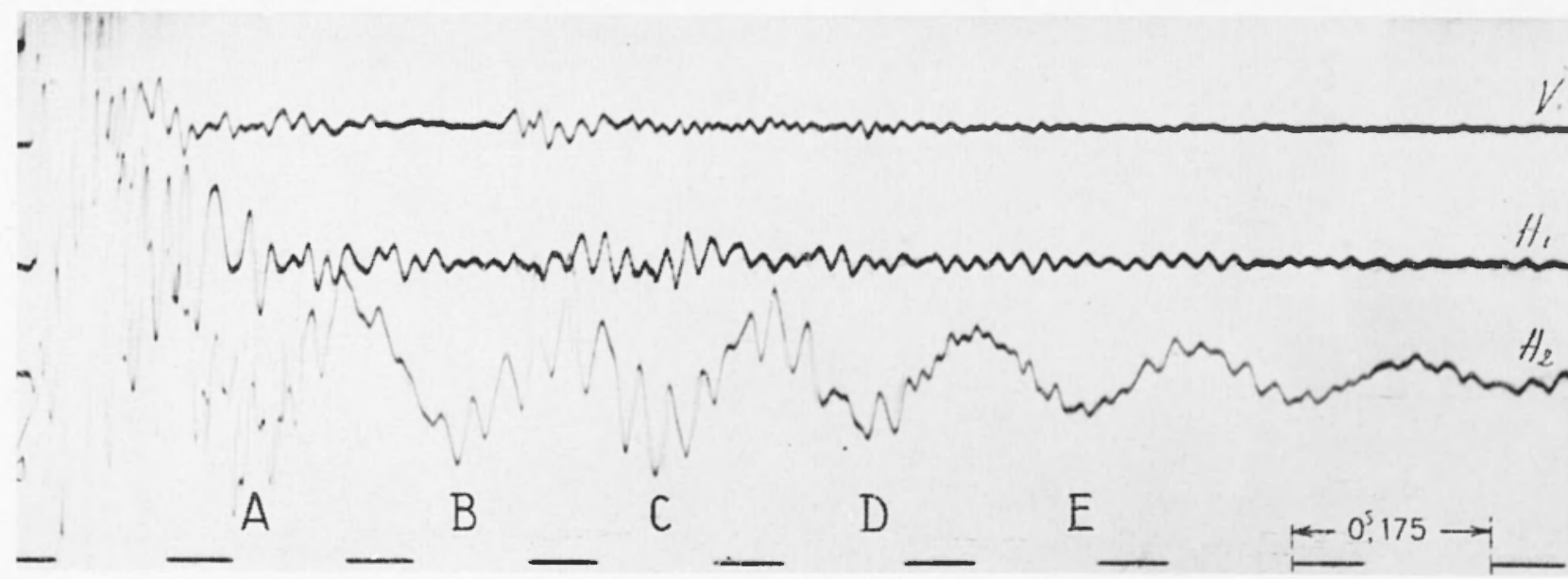

Fig. 3 - Oscillazioni con periodo di 0,2 see. circa $(\mathrm{AB}-\mathrm{BC}-\ldots)$ osservate nella valle del Cordevole. 

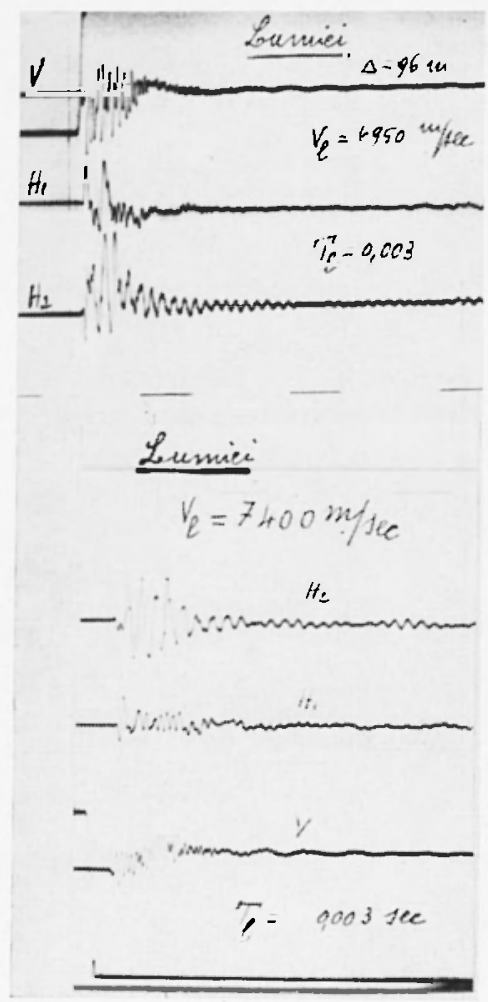

Fig. 4 - Registrazioni vibrografiche in roceia molto compatta, ad elevato modulo di elasticità (si noti l'elevatissima frequenza delle oscillazioni longitudinali o trasversali dirette).

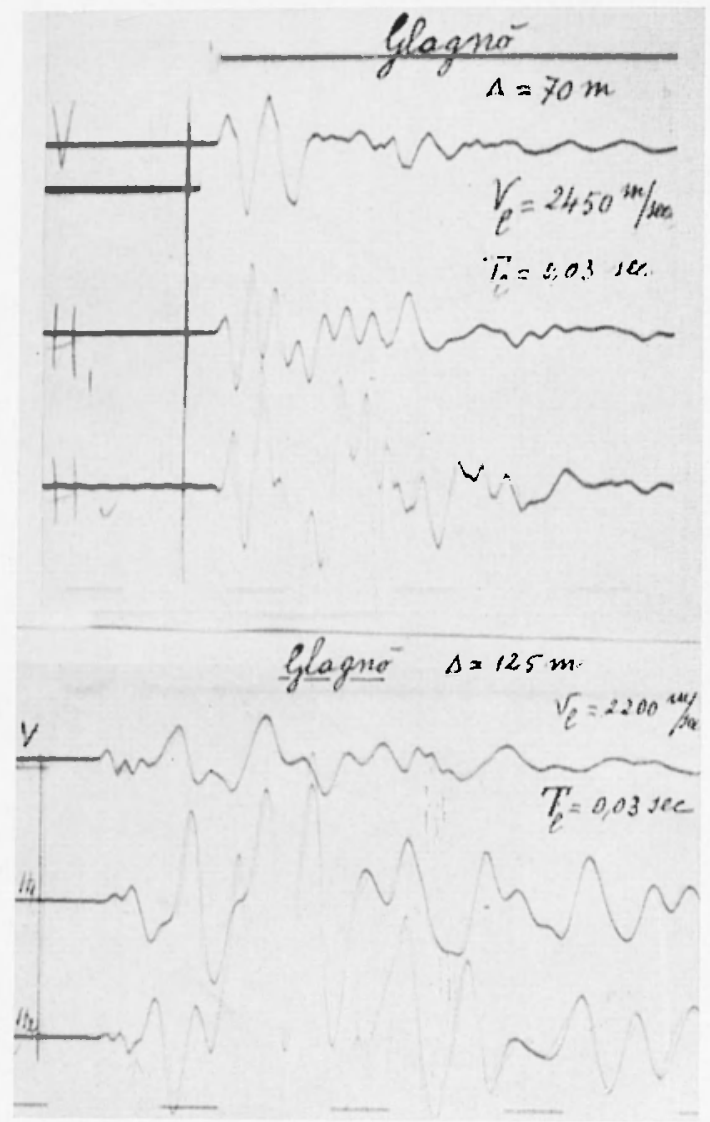

Fig. 5 - Registrazioni vibrografiche in roceia poco compatta, a basso modulo di elasticità (il periodo delle onde sismiche è notevolmente più alto di quello dolle oscillarioni, di cui alla fig. 4). 


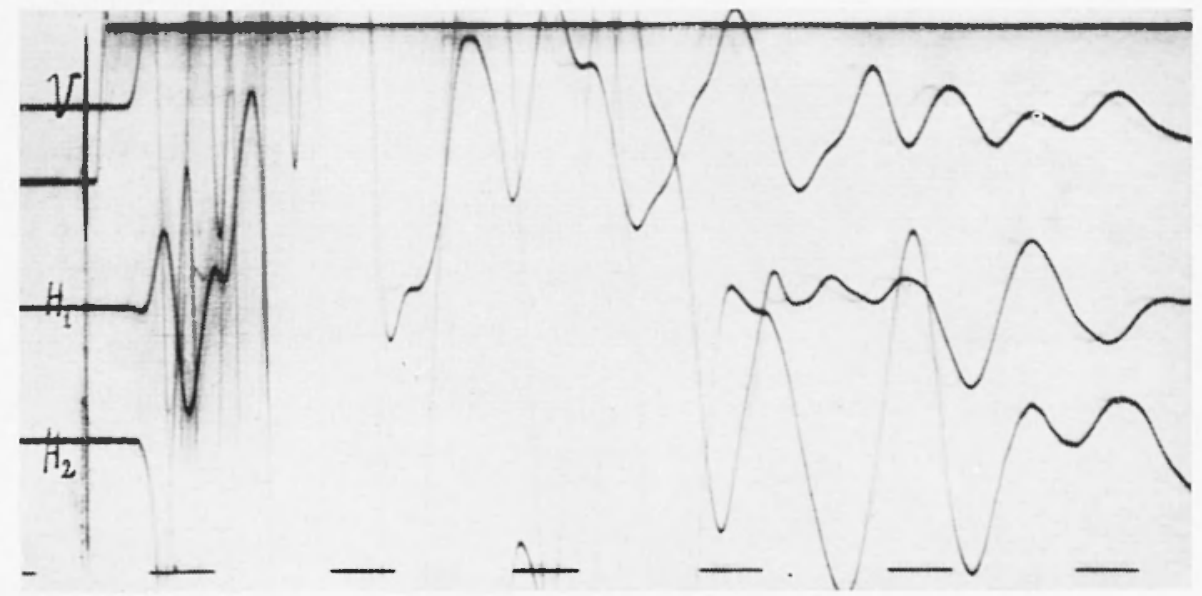

Fig. 6 - In terreno alluvionale, le oscillazioni presentano periodi elevati anche nelle onde longitudinali dirette $(0,06 \mathrm{sec}$. in questo esempio, tratto da esperienze nella valle del Gerlano, sopra una profonda coltre di seonnesso terreno di frana).

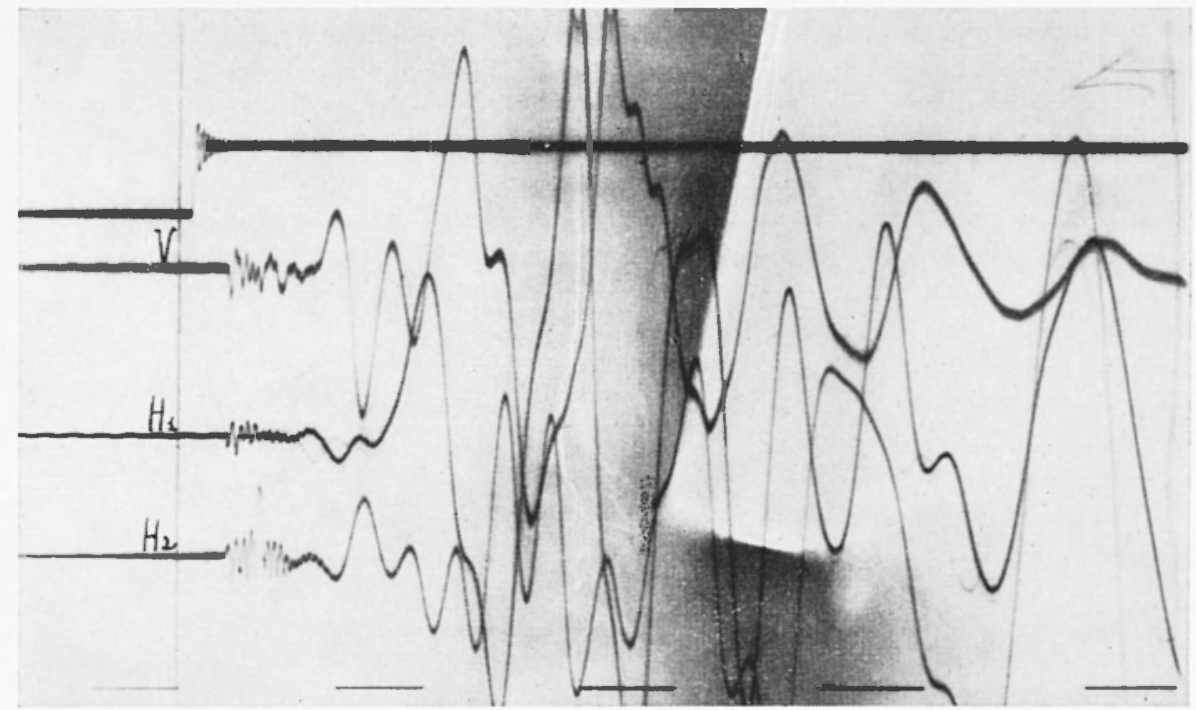

Fig. 7 - Registrazioni su terreno alluvionale, sovrastante a roccia: le fasi iniziali, di elevata frequenza, provengono dalla roceia sottostante (nell'esempio riportato, ottenuto a Ponte Noiariis, a circa 8 metri di profondita); le successive, proprie della coltre alluvionale, iniziano con periodi dellordine di 0,06 sec. 
Tav. W

P. CALOI
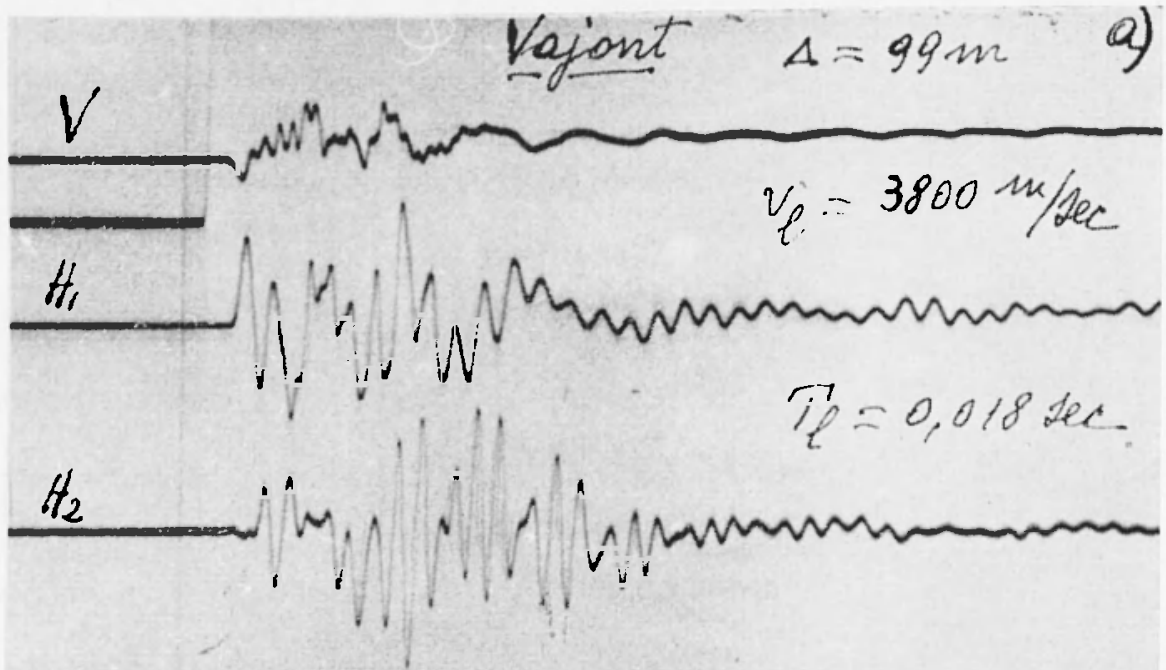

Vajont $A=49 \mathrm{~m}$ b)

$$
V_{l}=5900 \mathrm{~m} / \mathrm{sec}
$$

$H_{1}$

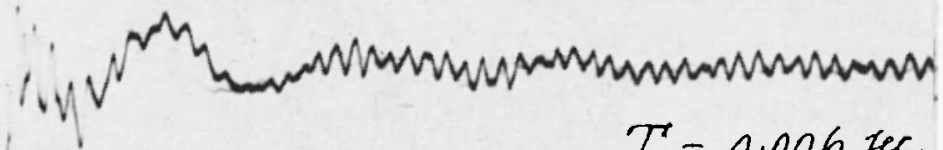

$$
T_{l}=0.006 \mathrm{sec}
$$

$H_{\text {s. }}$

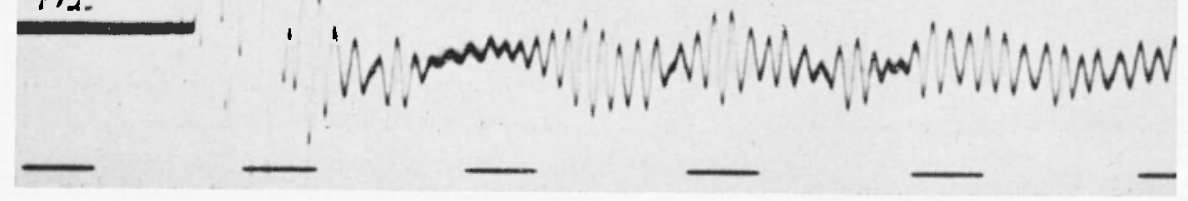

Fig. 8 - Zona roceiosa prima (a) e dopo (b) le iniezioni di cemento: il sensibile aumento della compattezza è rivelato sia dall ammentata velocita delle onde elastiche, sia dallammentata frequenza delle stesse. 
profondità, esso puó inviare in superficie, come onde rifratte, le oscillazioni che lo contraddistinguono, con più elevata frequenza. Tale contrasto risulta nettissimo dalla registrazione ottenuta in superficie, come appare chiaramente dai vibrogrammi della fig. 7 , ottenuti alla superficie di una coltre alluvionale, poggiante su rocecia alla profondità di 10 metri circa. Registrazione del genere sono sieura testimonianza che la roccia si trova a non grande profondità. Se lo spessore del materiale sciolto ì notevole, le onde rifratte dalla roceia sottostante vengono assorbite dal materiale incoerente (caso della fig. 6).

Il verificarsi della dispersione anomala nell'ambito delle alte frequenze, si presta ottimamente anche nelle operazioni di rigenerazione della roccia, quale controllo dell'efficacia delle iniezioni di cemento in roceia deterionata, indipendentemente da ogni nusura di velocitic. Vedansi p. es. le conseguenze dell'aumentata compattezza di un tratto di rocecia del Vajont, quale risulta dal confronto delle registrazioni fatte nello stesso mezzo prima e dopo l'iniezione del cemento (fig. 8).

Il tormento, derivante dall'azione dirompente dellesplosivo nellat fase di sbancamento della valle, seguito poi, a diga costruita, dal giuoco delle tensioni determinate nella zona di ancoraggio della diga, dalle flessioni in un senso o nell'altro - cui il manufatto viene sottoposto dalle variazioni dell'invaso e da quelle termiche - causa nella roceia un deterioramento del tessuto molecolare, che si traduce in un decadimento del modulo elastico. Ciò viene messo in chiara evidenza dalle registrazioni vibrogrufiche, sia quantitativamente, nella constatata variazione della velocità delle onde elastiche, sia qualitativamente, nel progressivo abbassarsi della frerpuenza d'onda, effetto del progressivo rilassamento del mezzo. Di ciò ho avuto modo di riferire a più riprese. Fenomeni strettamente collegati a quelli sopra accennati, si verificano nel caso di zone rocciose, soggette a pressioni crescenti, la cui azione può alfine provocare fratture a frane. Al crescere della pressione, nella fase iniziale, può addirittura constatarsi un aumento nella velocità di propagazione delle onde elastiche. Ciò probabilmente è da attribuirsi alla riduzione o alla scomparsa degli spazi vuoti, preesistenti fra cristallo e cristallo, in seguito alla compressione. Quando però quest'ultima comincia ad intaccare la compattezza dell'edificio molecolare, facendo passare il mezzo dal campo elastico a quello plastico, allora la velocita di propagazione delle onde sismiche comincia a decrescere. La diminuzione si mantiene limitata. Ma se la pressione, cui la rocecia è sottoposta, persiste a lungo o si arcresce, si giunge al limite di frattura, in corrispondenza del quale la velocità subisce un improviso collasso. Nella fase plastica, la lieve dimi- 
nuzione di velocità de da attribuire alla formazione di sottili fenditure con dislocazione di cristalli, in corrente plastica. Tella fase di rottura, le fenditure si ingrandiscono in larghe fratture, con annientamento dell'edificio molecolare. Casi del genere, sono stati seguiti in zone dove era manifesta l'azione preponderante di strati sovrastanti, con sintoni di cedimento della roceia sottoposta a pressione; cedimento mutatosi al line in moto franoso.

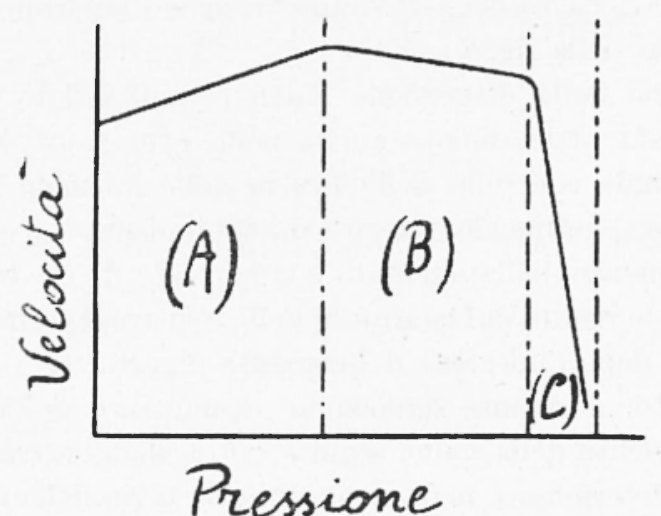

Fig. 9 - (A) fase elastica, (B) fase plastica, (C) fave di frattura (secondo Shimozuru).

L'andamento del fenomeno, cosi come è stato descritto ha trovato conferma in esperienze di laboratorio ( $\left.{ }^{21}\right)$. La fig. 9 sintetizza l'andamento degli esperimenti, compiuti su campioni di mamo.

2. - Nelle numerose esperienze di registrazione di onde elastiche, compinte presso bacini idrici, ho avuto modo di osservare un altro interessante fenomeno, che qui intendo solo accennare. Più volte ho disposto le esperienze cosi da ottenere la registrazione, su un lato della valle, di esplosioni provocate sul lato opposto. Le esperienze sono state compinte sia a valle della diga che a monte, in corrispondenza del bacino pieno. Ebbene, invariabilmente, mentre a valle della diga le onde longitudinali venivano largamente registrate come onde diffratte (fig. 10), in corrispondenza del fondo valle, a monte della diga le onde longitudinali diffratte praticamente mancavano. Esse erano invece seguite da ampie oscillazioni, che avevano attraversato il bacino idrico con la velocita delle onde sonore. Si verifica quindi un pressoché totale trasferimento di energia elastica dal mezzo solido a quello liquido. A conferma di tale tra- 


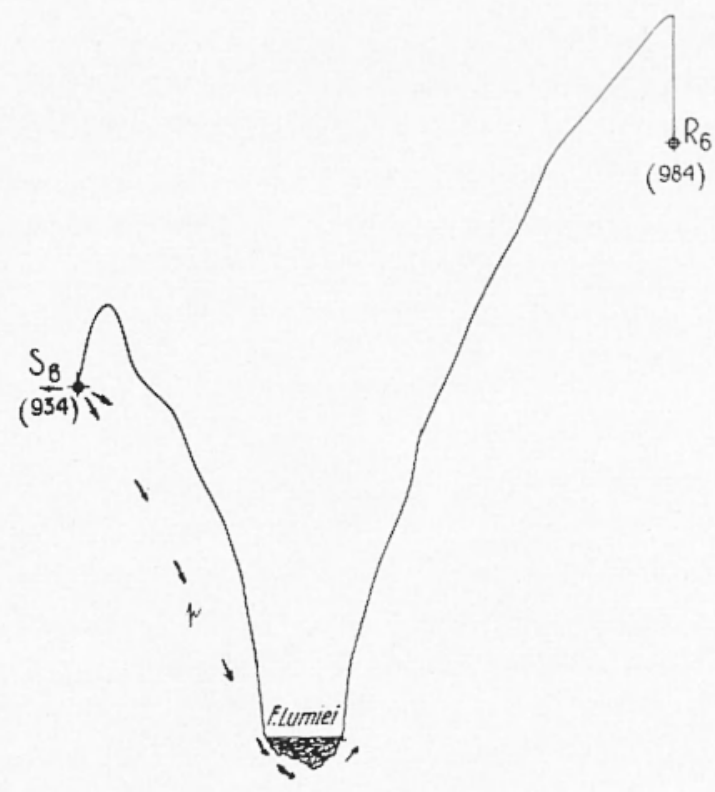

a)

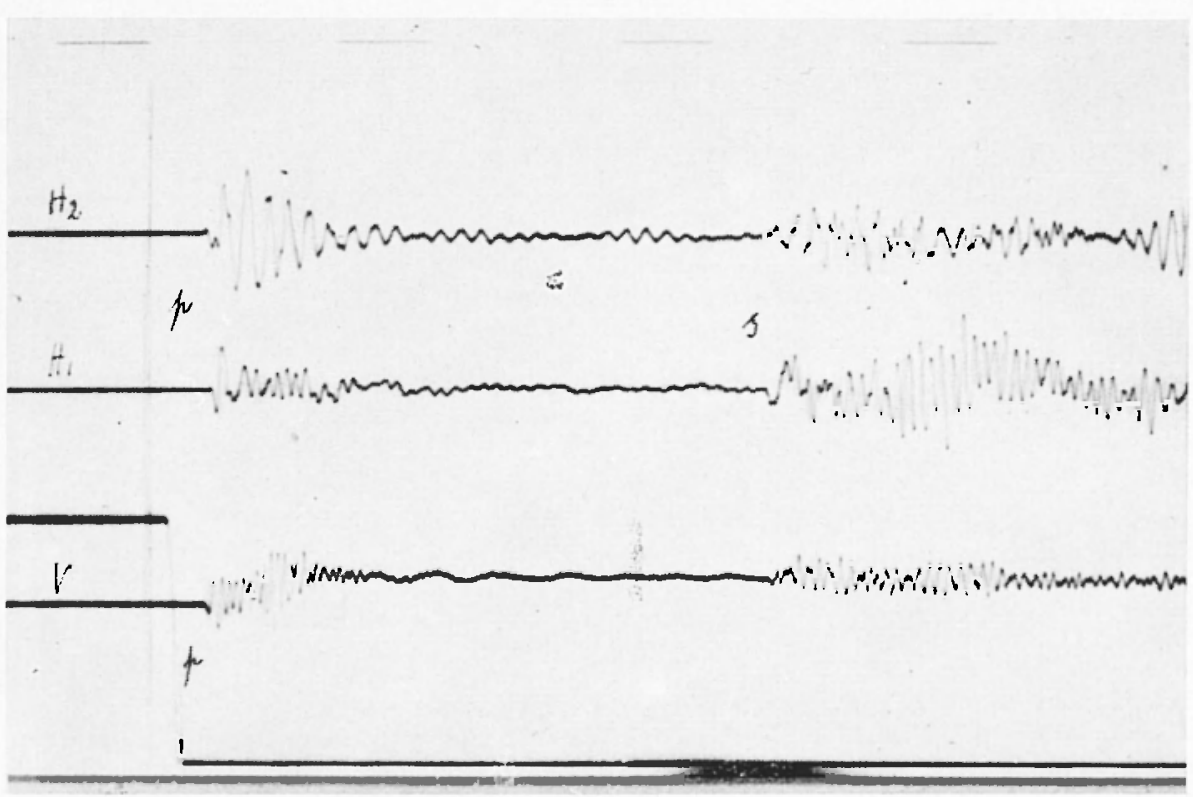

Fig. $10-a)$ Scoppio provocato in $S_{6}$, da un lato della valle del Isumiei, e registrato in $P_{6}$ sul lato opposto; $b$ ) le onde $p$ (diffratte-riflesse) risultano nette ed ampie ( $s$, onde di pressione). 
TAV. VI

P. CALOI

a)
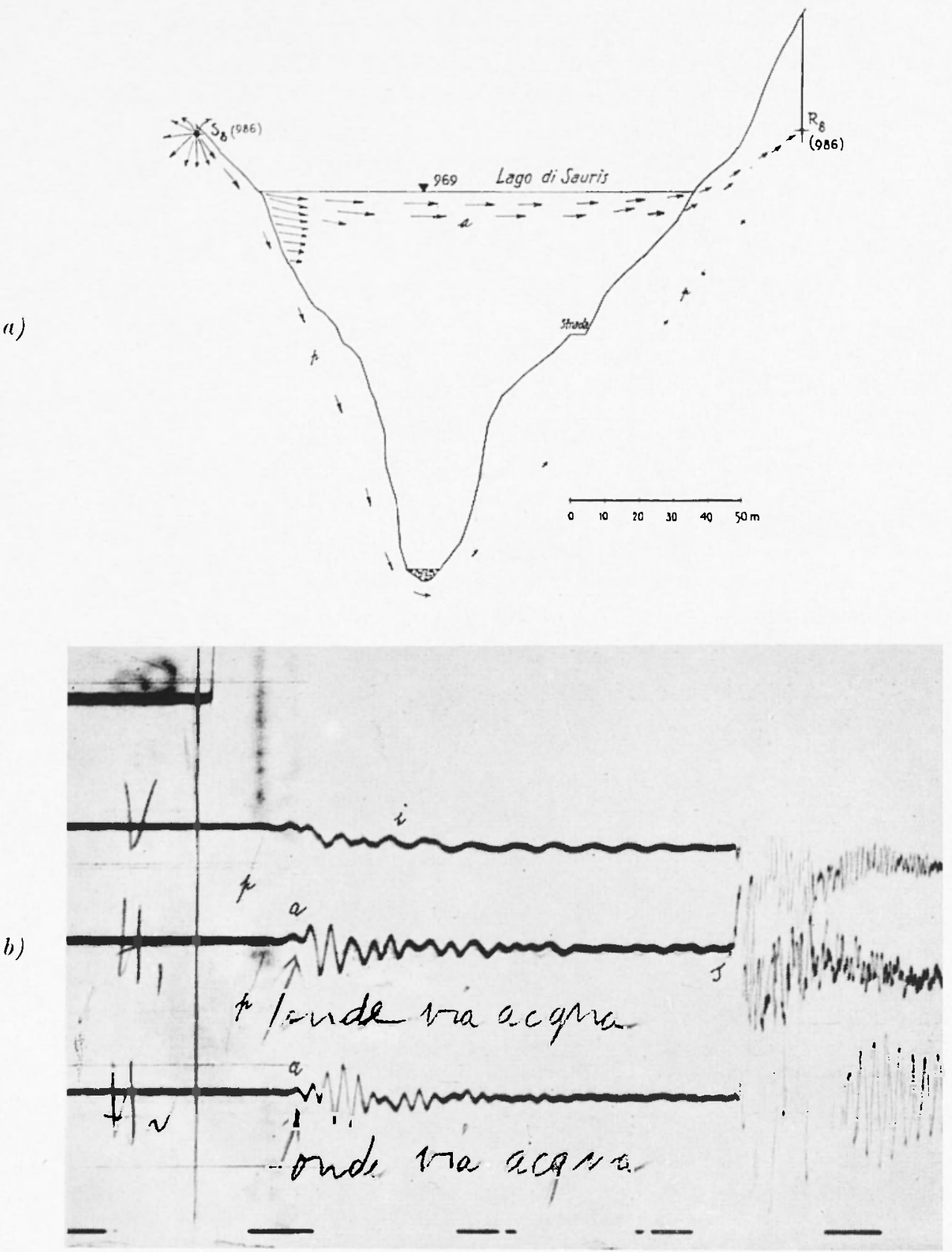

Fig. 11 - a) Registrazione a monte della diga del Lumiei: punto di scoppio in $S_{R}$, punto di registrazione in $R_{8}$, sull altra sponda del lago;

b) le onde $p$ sono di ampiezza trascurabile; vengono inveco registrate chiaramente le onde a che hamno attraversato il lago, con la velocitì di $1450 \mathrm{~m} / \mathrm{see}$ ea. 

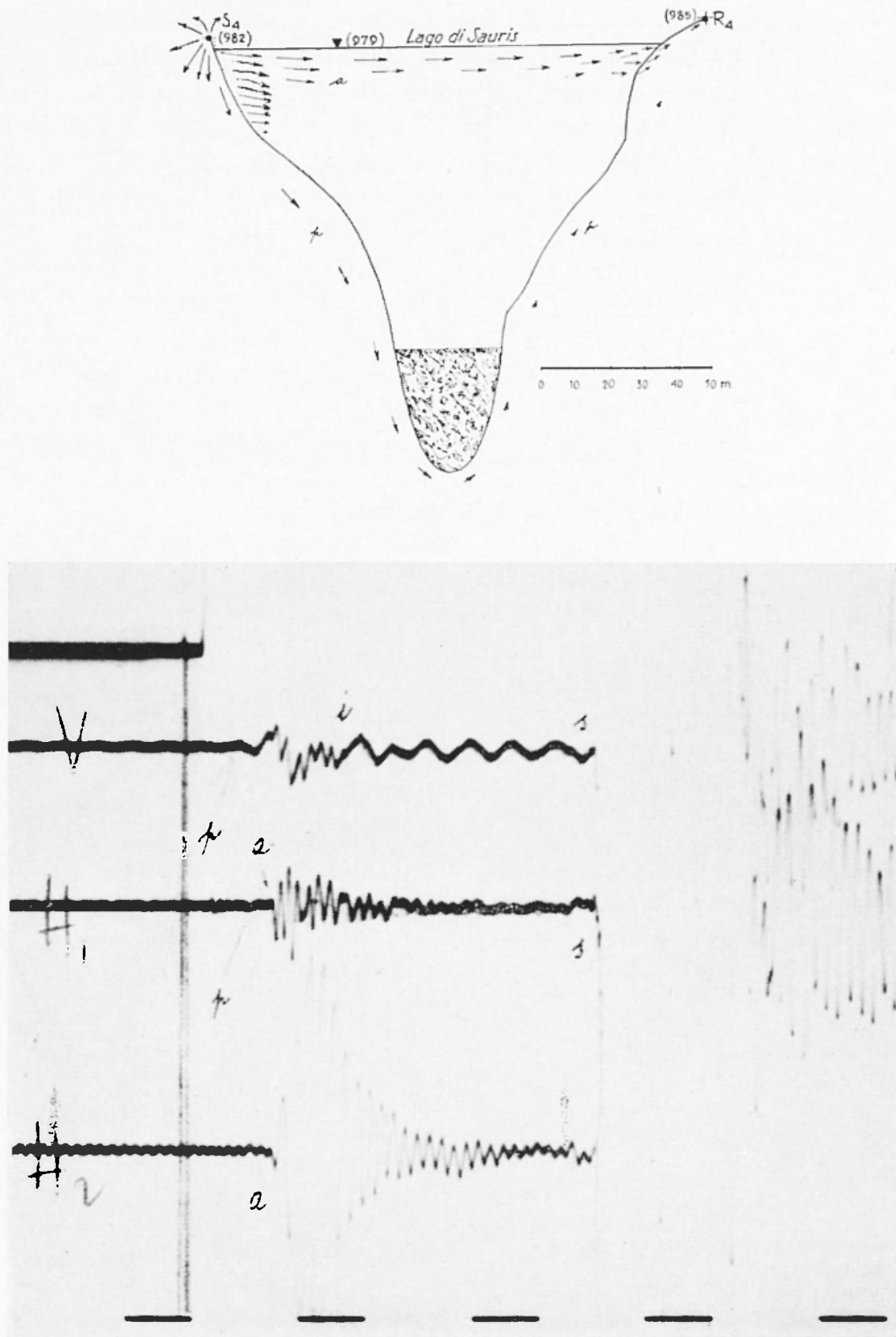

Fig. 12 - a) Scoppio in $S_{4}$, registrazione in $R_{4}$ sull'altra sponda del lago; b) lieve ampiezza delle onde $p$, mentre risultano marcatissime le onde a che hanno attraversato il bacino idrico, subendo due rifrazioni successive. 
TAV. VIII

a)

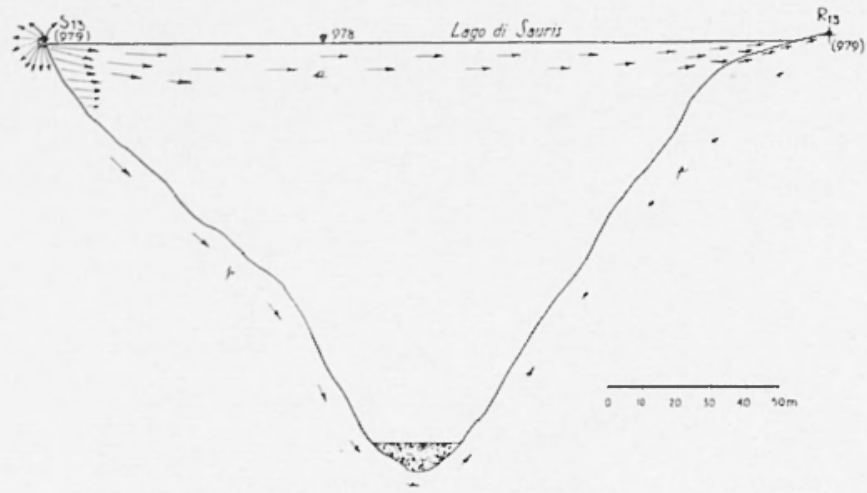

b)

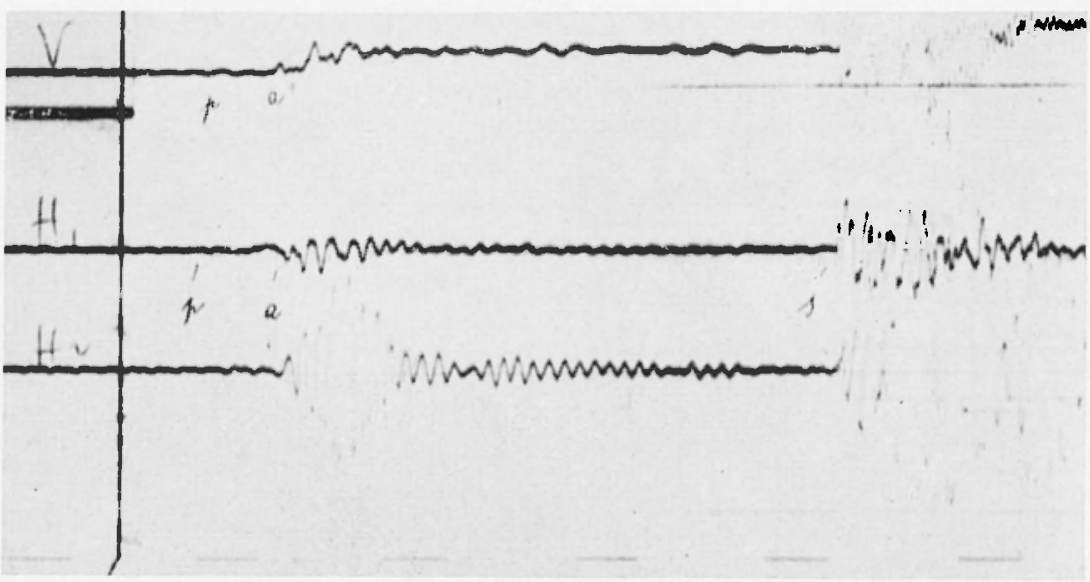

c)
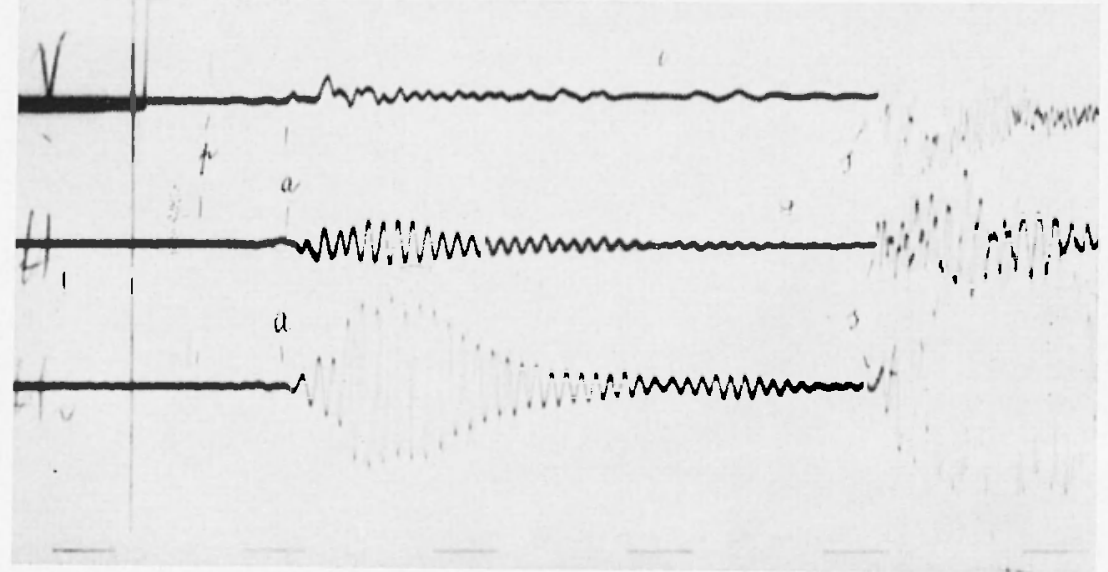

Fig. 13 - a) Scoppio in $S_{13}$, registrazione in $R_{13}$, sull'altra sponda del lago; b) onde diffratte-riflesse di ampiezza trascurabile, ampie onde $a$ attraversanti il lago con velocità dell'ordine di $1450 \mathrm{~m} / \mathrm{sec}$.

c) registrazioni su tragitto analogo. 
sferimento sta non solo il fatto della pressoché totale scomparsa di onde longitudinali diffratte, condotte dal mezzo solido, ma altresì l'ampiezza delle oscillazioni che hanno attraversato il lago, malgrado due rifmazioni intermedie, fra solido e liquido e viceversa (figg. 11-15). Tale trasferimento di energia sembra scostarsi sensibilmente dalle leggi della riflessione-rifrazione fra mezzi solido e liquido a contatto. Può darsi sia specifico delle elevate frequenze. E caratteristica poi la formazione di onde nate dall'accoppiamento acqua-aria, che precedono l'arrivo dell'onda di pressione o che appaiono particolarmente chiare sulla componente verticale.

L'energia trasmessa all'acqua, una volta ritrasmessa al mezzo solido, subisce in esso una rapida estinzione; a questo riguardo, sono significativi gli esempi forniti dalle registrazioni, di cui alle figure 12 e 11. La prima è stata ottenuta a pochi metri dalla riva, mentre la seconda si è avuta a $30 \mathrm{~m}$ circa dal margine del lago.

Il trasferimento dell'energia elastica al bacino idrico e la trasmissione da parte di quest'ultimo, vengono a creare per la diga condizioni di asimmetria dinamica, in caso di scosse locali, legate alle sollecitazioni che la diga stessa deve sopportare da parte del lago.

Mi riservo di riprendere l'argomento, anche nei suoi aspetti sismologici.

3. - Ho già detto estesamente altrove della grande utilità del vibrometro nella determinazione del modulo elastico del mezzo in esame. Ho sottolineato che tale utilità si rende particolarmente manifesta nella possibilità di prescindere, con tale strumento, dalla conoscenza del rapporto di Poisson, che -- come è noto - non è affatto costante, pur variando entro limiti generalmente non molto discosti. La contemporanea registrazione delle onde trasversali, permette infatti di calcolare il modulo di Young $E$ con la formula:

$$
E=\varrho \frac{v_{2}{ }^{2}\left|3\left(\frac{v_{1}}{v_{2}}\right)^{2}-4\right|}{\left(\frac{v_{1}}{v_{2}}\right)^{2}-1},
$$

dove $o$ è la densità, $v_{1}$ la velocità delle onde longitudinali e $v_{2}$ quella delle onde trasversali, propric del mezzo.

L'uso del vibrometro diviene di grande interesse anche nel controllo delle caratteristiche dinamiche (e quindi elastiche) di una diga. Le variazioni di tali caratteristiche possono dedursi non solo mediante la determinazione diretta del modulo di Young del calcestruzzo, ma altresì regi- 
strando, di quando in quando, le oscillazioni libere trasversali dei conci della diga. I periodi di queste oscillazioni sono legati al modulo $E$ e alla densità o del calcestruzzo, attraverso la formula $\left({ }^{16}\right)$ :

$$
T=\frac{4 \pi \mid \overline{3}}{\vartheta^{2}} \cdot \frac{l^{2}}{b} \cdot \frac{1}{|E|_{\varrho}}
$$

dove $l$ è l'altezza del concio prescelto, $b$ il suo spessore medio, $|\bar{E}|_{2}$ la velocità delle onde longitudinali nel calcestruzzo (come corpo finito, in forma di sbarra), mentre $\theta$ (legato, fra l'altro, ad $l$ e alla pulsazione delle onde stazionarie), consegue come una delle radici dell'equazione

$$
\cosh \theta+\cos \theta+1=0 \text {. }
$$

Eventuali variazioni nel tempo subite dalla grandezza $T$ testimoniano di variazioni nelle caratteristiche fisiche del calcestruzzo; queste possono dipendere sia da variazioni nella rigidita del calcestruzzo, sia dall'intervento di lesioni.

\begin{tabular}{|c|c|c|c|c|c|c|}
\hline & \multicolumn{2}{|c|}{ Lninodali } & \multicolumn{2}{|c|}{ Binodali } & \multicolumn{2}{|c|}{ Trinodali } \\
\hline & calc. & oss. & calc. & oss. & calc. & oss. \\
\hline $\begin{array}{l}\text { Diga di Pieve di Cadore. . } \\
\text { (concio XIV) }\end{array}$ & 0,23 & 0,21 & - & -- & - & - \\
\hline $\begin{array}{l}\text { Diga del Lumiei. } \ldots \\
\quad \text { (concio centrale) }\end{array}$ & -- & - & 0,25 & 0,23 & 0,10 & 0,10 \\
\hline $\begin{array}{l}\text { Diga di Val Gallina. . . . } \\
\text { (concio centrale) }\end{array}$ & - & - & 0,13 & 0,15 & - & - \\
\hline Diga di Barcis & - & - & 0,10 & 0,11 & - & - \\
\hline
\end{tabular}

'Tabella - Periodo oscillazioni stazionarie trasversali.

La tabellina riporta i risultati di osservazioni e di calcoli, discussi nelle note citate, relativi a dighe della Società Adriatica di Elettricità.

Le figure 16-20 danno esempi di registrazioni ottenuti su varie dighe.

Di non minore interesse risulta l'uso di stazioni sismiche presso le grandi dighe. Anche su questo argomento ebbi a intrattenermi a lungo, a più riprese $(3,7,10,12,17,20,22)$. 


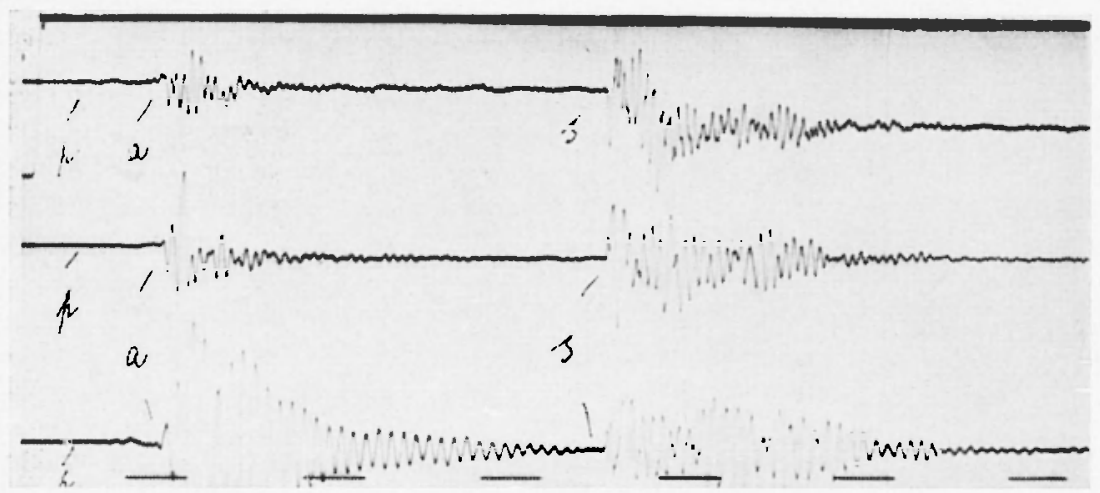

Fig. $14-a)$ Scoppio in $S_{13}$, registrazione in $\kappa_{13}$ sull altra sponda del lago; b) onde $p$ trascurabili, anpie le a che hanno attraversato l'elemento liquiuilo.
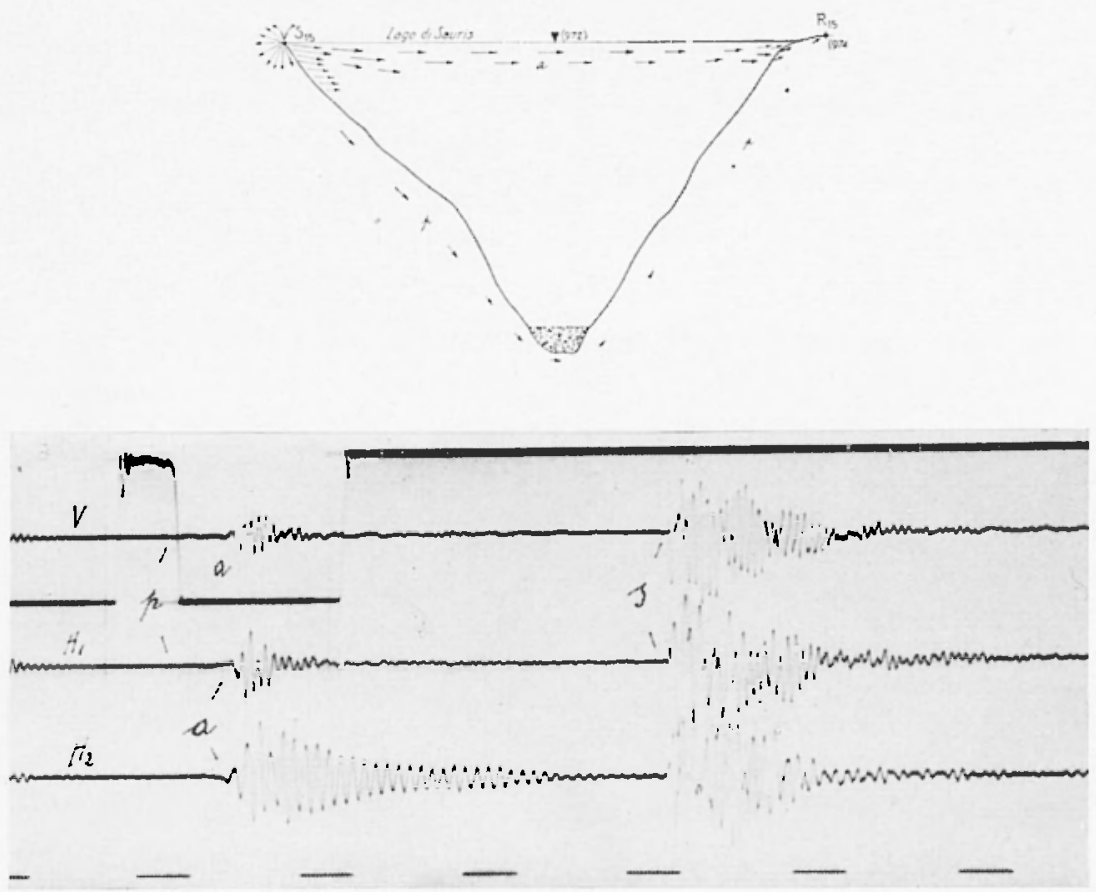

Fig. $15-a)$ scoppio in $R_{15}$, registrazione in $R_{15}$, sullopposta sponda del lago; b) onde $p$ appena accennate, anpie onde a cui è associata un alta percentuale dell'energia sviluppata in $S_{15}$. 


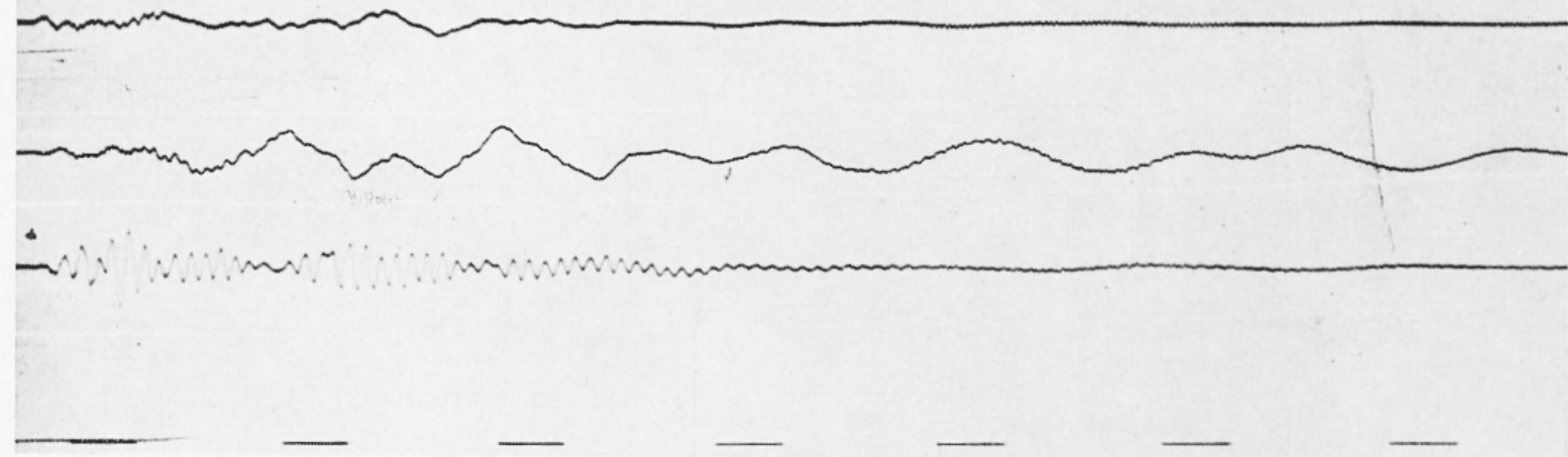

Fig. 16 - Vibrazioni libere binodali e uninodali (vibrogramma centrale) della diga di Pieve di Cadore (concio XIV).

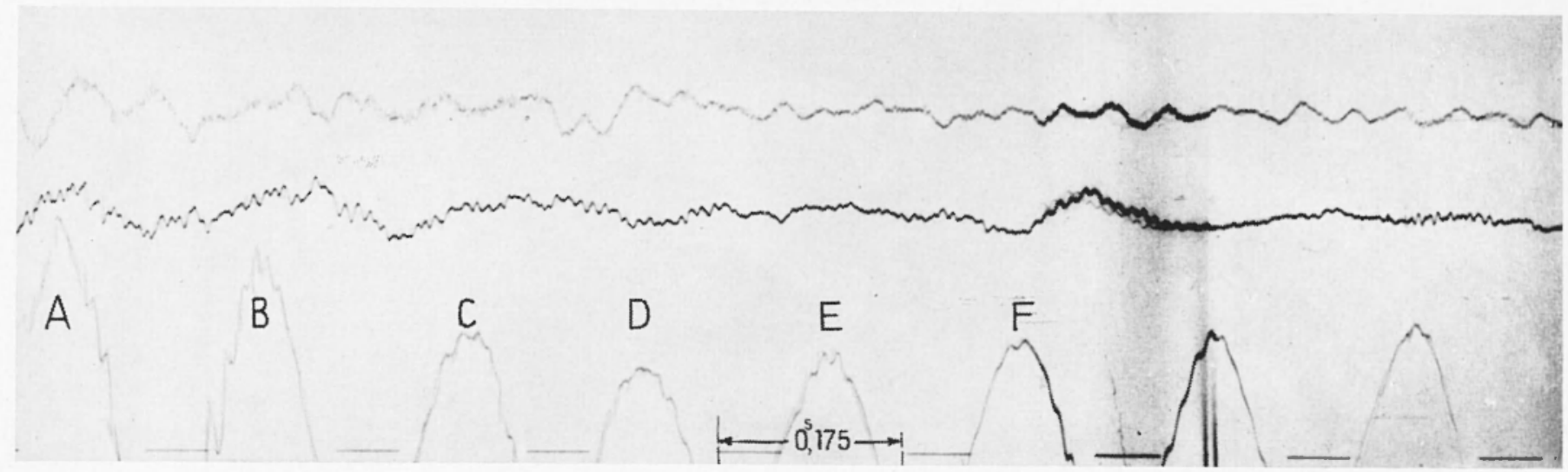

Fig. 17 - Vibrazioni libere binodali $(\mathrm{AB},-\mathrm{BC}, \ldots)$ trasversali della diga del Jumiei (1954), sulla comp. valle-monte $\mathrm{H}_{2}$. 


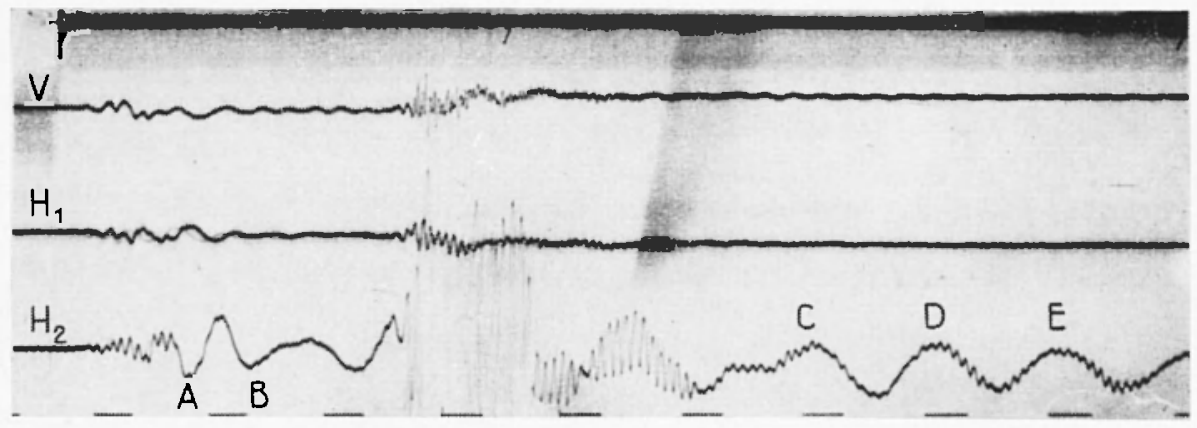

Fiæ. 18 - Vibrazioni libere trasversali trinodali (AB) e binodali (CI) della diga di Val Gallina (1953).

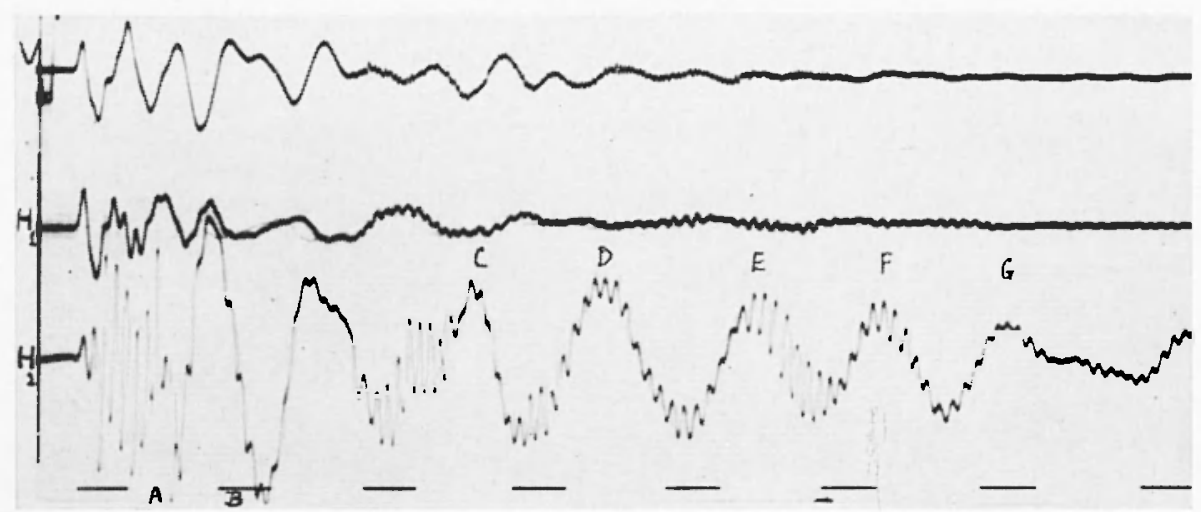

Fig. 19 - Vibrazioni libere trasversali (AB) e binodali (CD) della diga di Val Gallina (1955).

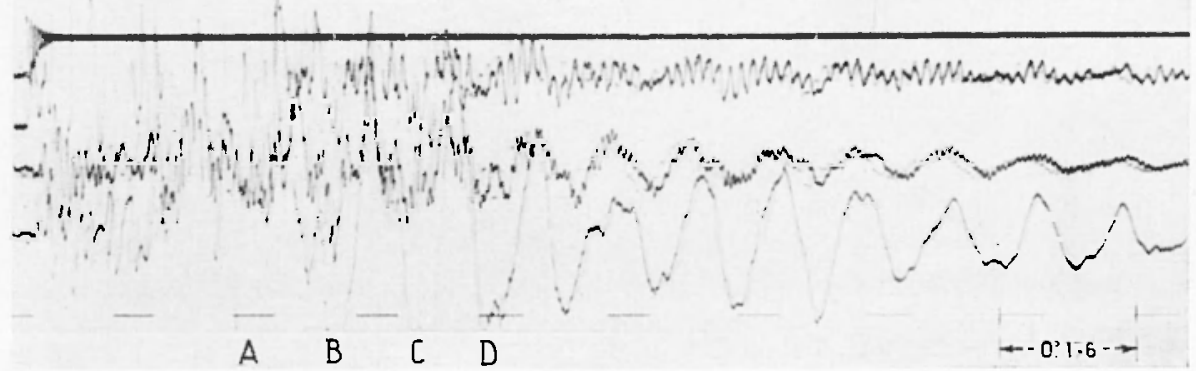

Fin. 20 - Vibrazioni libere trasversali binodali (AB, BC) .. della diga di Bareis (1959). 


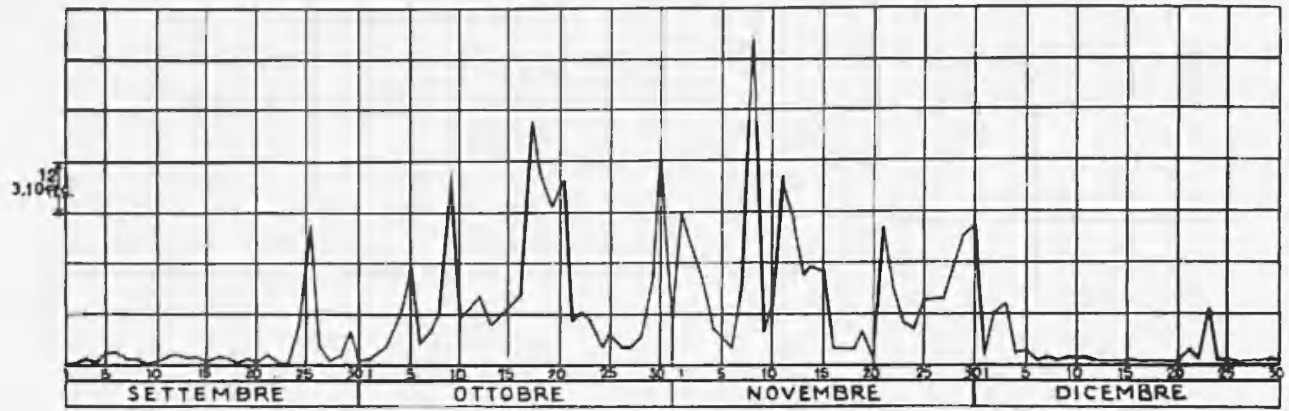

Fig. 21 - Energia (in $3 \cdot 10^{12}$ erg) associata alle microscosse provocate dalla flessione autumale verso valle della diga di Pieve di carlore, nell autu11110 195).

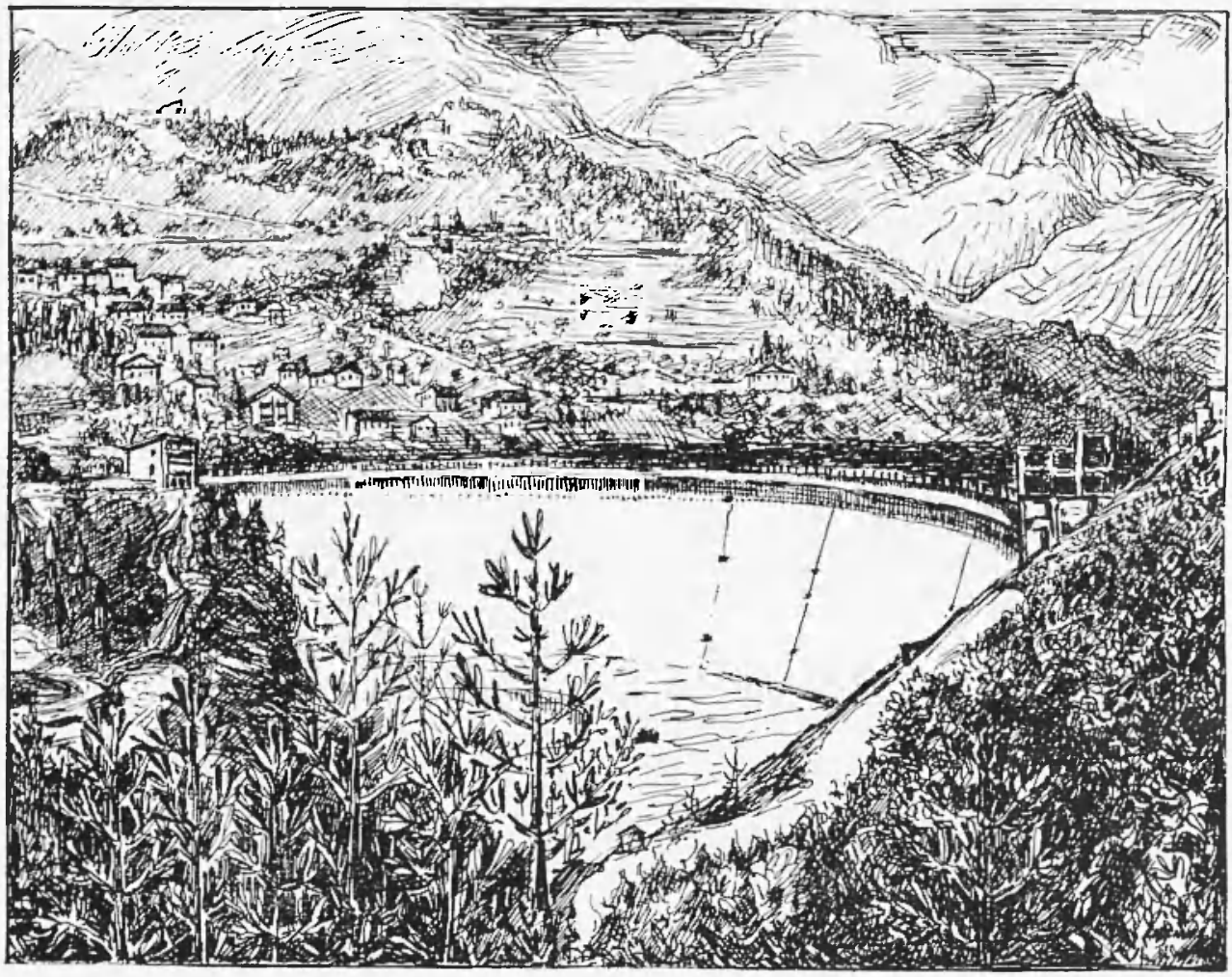

Fig. 21 bis - Diga di Pieve di Cadore (disegno di Luciana Luciani) nella quale funzionano diverse coppie di fotoclinografi; nello scantinato della cabina-comandi (a sinistra della figura) funziona una stazione sixmica, costituita da una terna di apparecchi elettromagnetici. 
Particolarmente prezioso riesce l'uso del sismografo nel periodo che va dallo sbancamento ai primi anni dopo la costruzione della diga. L'equilibrio delle tensioni elastiche risulta, in questo periodo, - sia pure superficialmente - rotto: di qui tutta una serie di microterremoti, che accompagnano il lavorio dinamico della roccia, tesa verso nuove posizioni di equilibrio. E in questa fase di crisi che le zone rocciose più esposte al giuoco delle tensioni subiscono sensibili flessioni nelle cartteristiche elastiche, rivelate dal decadere del modulo. I alle registrazioni delle stazioni sismiche si può risalire all'ordine di grandezza dell'energia associata alle singole mieroscosse.

Con sufficiente approssimazione, l'energia $E$ associata ad una componente di una scossetta può essere cosi espressa ( ${ }^{3} .{ }^{7}$ )

$$
E=4 \pi^{3} \varrho v t\left(\frac{a}{T}\right)^{2} \Delta^{2} \quad, \quad \text { c.g.s. }
$$

dove $o$ è la densità della roceia, $v$ la velocità delle onde longitudinali (o trasversali) prese in esame, $t$ la durata in secondi del treno d'onde di periodo $T$ ed ampiezza media $a$, registrato alla distanza $\triangle$.

I'energia associata alle altre due componenti può ritenersi all'incircar uguale.

La distanza origine del movimento può essere facilmente dedotta dalla registrazione delle onde longitudinali $(p)$ e trasversali $(s)$. Determinate con esperienze preliminari, le velocità medie di propagazione delle onde longitudinali $v_{p}$ e trasversali $v_{s}$, proprie della roceia, nella zona della diga, si ha - se $s-p$ indica la differenza nei tempi di arrivo fra le onde trasversali e longitudinali -

$$
\Delta=\frac{v_{p} \cdot v_{s}}{v_{p}-v_{s}}(s-p) .
$$

Le microagitazioni, legate al tormento delle flessioni della diga nella roccia d'imposta, hanno un particolare aspetto $\left({ }^{10}\right)$ e si registrano numerose nelle epoche in cui la diga compie i suoi movimenti stagionali monte-valle e viceversa. I a fig. 21 dà l'ammontare dell'energia associata alle mieroscosse, registrate presso la diga di Pieve di Cadore nel periodo Settembre-Dicembre 1951. Lienergia liberata tende a crescere verso la metà di Ottobre e laggiunge il suo massimo a mezzo Novembre; quindi gradualmente decresce e si annulla nella prima decade di Diembre. In questo periodo, comè noto, la diga esegue la graduale flessione verso valle - in concomitanza col progressivo diminuire della temperatura dell'aria -, che presenta la sua acuta accentuazione nei mesi di Ottobre 
e Novembre. Ciò è provato, del resto, dalle contemporanee registrazioni clinogratiche, effettuate nei conci, e nei pressi, della diga.

La crisi geodinamica nella zona di una diga dipende da svariati fattori: natura della roceia d'imposta, omogeneità od eterogeneità di questa, variazione continua o meno delle sue caratteristiche elastiche, adattabilità del manufatto all'ambiente fisico e bontà della sua realizzazione, ece. Nella zona interessata dalla diga di Pieve di Cadore, l'attività microsismica è durata, nel suo complesso, circa sei anni: iniziatasi con le operazioni di sbancamento (19-8), ha presentato i suoi massimi nel biemio 1950-1952, per esaurirsi nel 1955.

Le figg. 22 e 23 riportano esempi di microscosse, registrate presso la diga di Pieve nel periorlo sopra accemnato.

La reazione elastica della roccia, sorpresa nelle sue condizioni di equilibrio naturale, varia da situazione a situazione. Ho accennato, nel precedente lavoro $\left({ }^{22}\right)$, al caso del Vajont e alle vicissiturlini che riportarono le fiancate della valle verso un nuovo equilibrio, dopo la complessa inquietudine, insorta con i lavori di sbancamento. Anche dal punto di vista sismico, la stretta del Vajont presenta aspetti che la distinguono da quella di Pieve di Cadore. La stazione sismica in fumzione al Vajont, unitamente a quelle di Pieve di Cadore e di Tolmezzo, da un contributo preziosissimo alla conoscenza sismo-tettonica della zona, specie per quanto si riferisce al meceanismo dei piecoli terremoti che la interessano (v. figg. 24 e 25 ).

4. - A più riprese ho avuto modo di sottolineare, con testimonianze, l'eccezionale interesse del clinografo, sia nella geofisioa generale che in quella applicata. Naturalmente, eviterò di ripetermi.

Il clinografo in funzione in numerose località del Cadore e della Carnia è retto dall'equazione

$$
I \frac{d^{2} \varphi}{d t^{2}}+\left(\frac{2}{3} l m g i+\frac{\pi r^{4} \mu}{\lambda}\right) \varphi=0,
$$

dove $\varphi$ è l'angolo di eni ruota il pendolo bifilare per una variazione $\psi$ della verticale apparente, $I$ è il momento d'inerzia della massa $m$ rispetto all'asse di rotazione, $l$ la lunghezza della sbarretta portante la massa, g l'accelelazione di gravità, $i$ l'angolo d'inclinazione dell'asse del pendolo sulla verticale, $r$ il raggio dei fili di sospensione, di lunghezaa media $\lambda$ e di rigidità $\mu$. Il coefficiente di $\varphi$, diviso per $I$, dà il quadrato della pulsazione, donde l'espressione del periodo. 

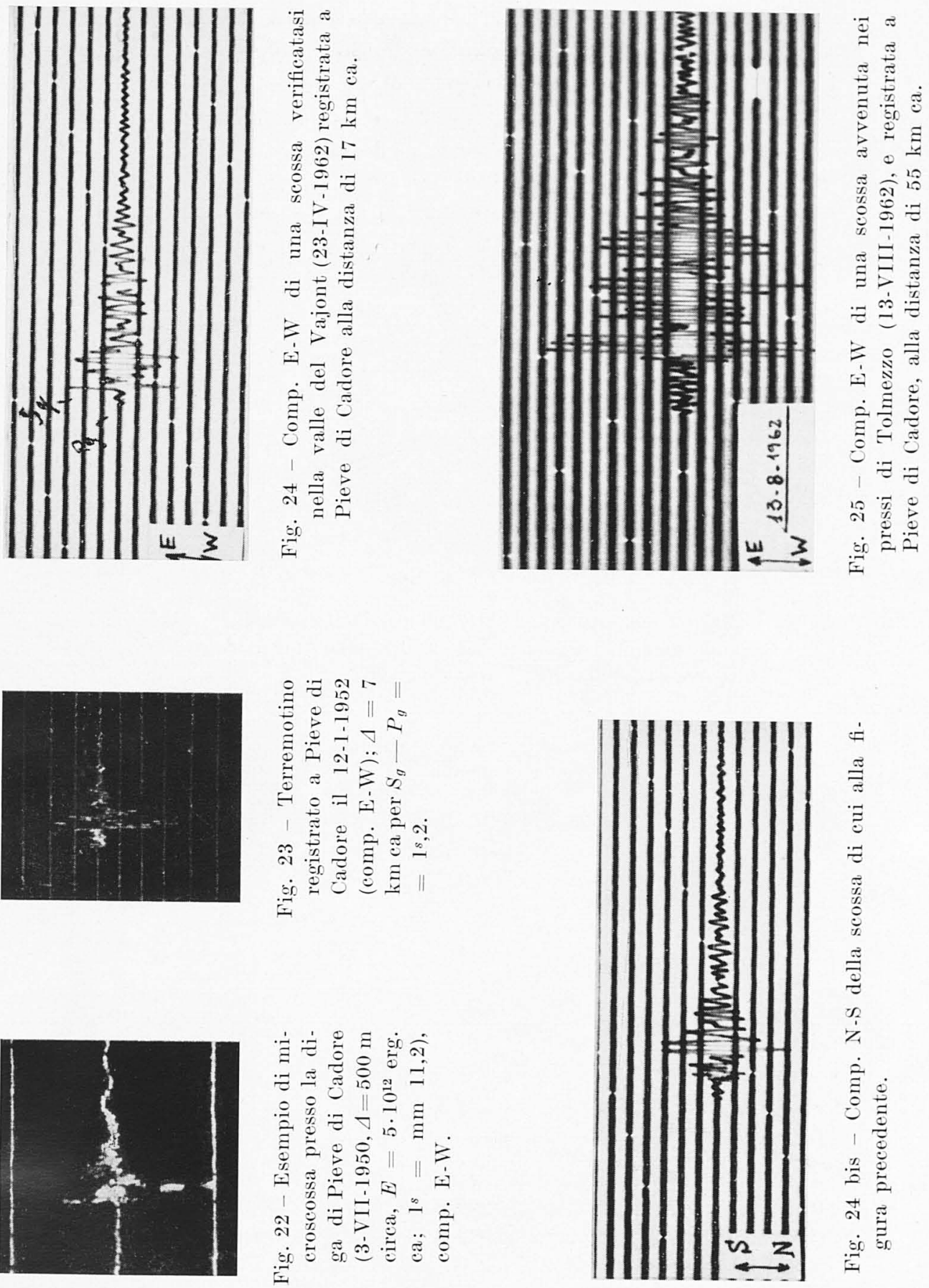

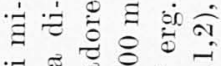

च

我:

छ

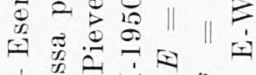

党

ช. 
8

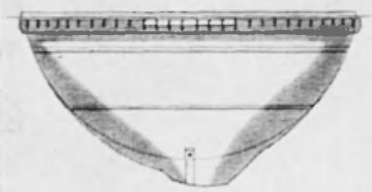

11

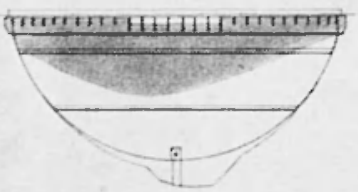

Jcala $\int_{430}^{400}$
9

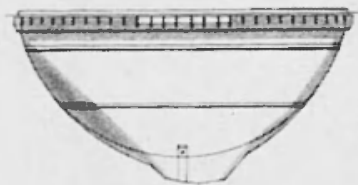

12

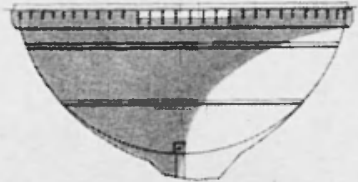

14

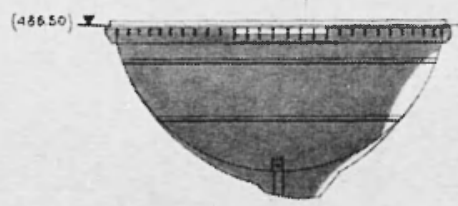

10

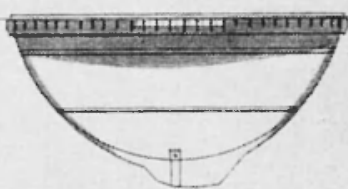

13

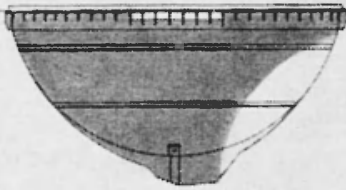

$3 \cdot 71 \cdot 1958$

Fig. 29 - Diga dell'Ambiesta. Andamento dell'insolazione nei primi di Giugno (secondo Giovanni De Vido): essa interessa principalmente il lato sinistro della diga (vista da valle).

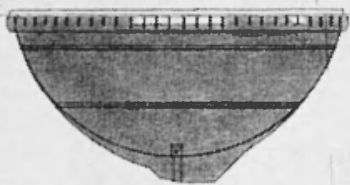

10.30

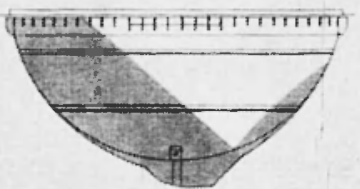

Seala $\begin{aligned} & 190 \\ & 480 \\ & 470 \\ & 450 \\ & 450 \\ & 440 \\ & 450\end{aligned}$

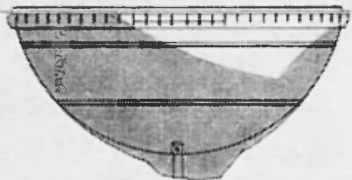

12

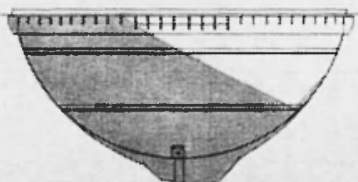

15

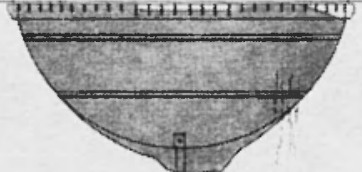

10

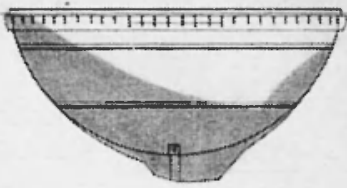

14

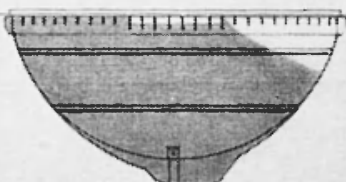

$15 \cdot x_{1} \cdot 1957$

Fig, 30 - Diga dell'Ambiesta. Andamento dell'insolazione a metà Novembre (secondo Giovanni De Vido): conferma dell'asimmetria dell'inso. lazione, prevalente sul lato sinistro. 

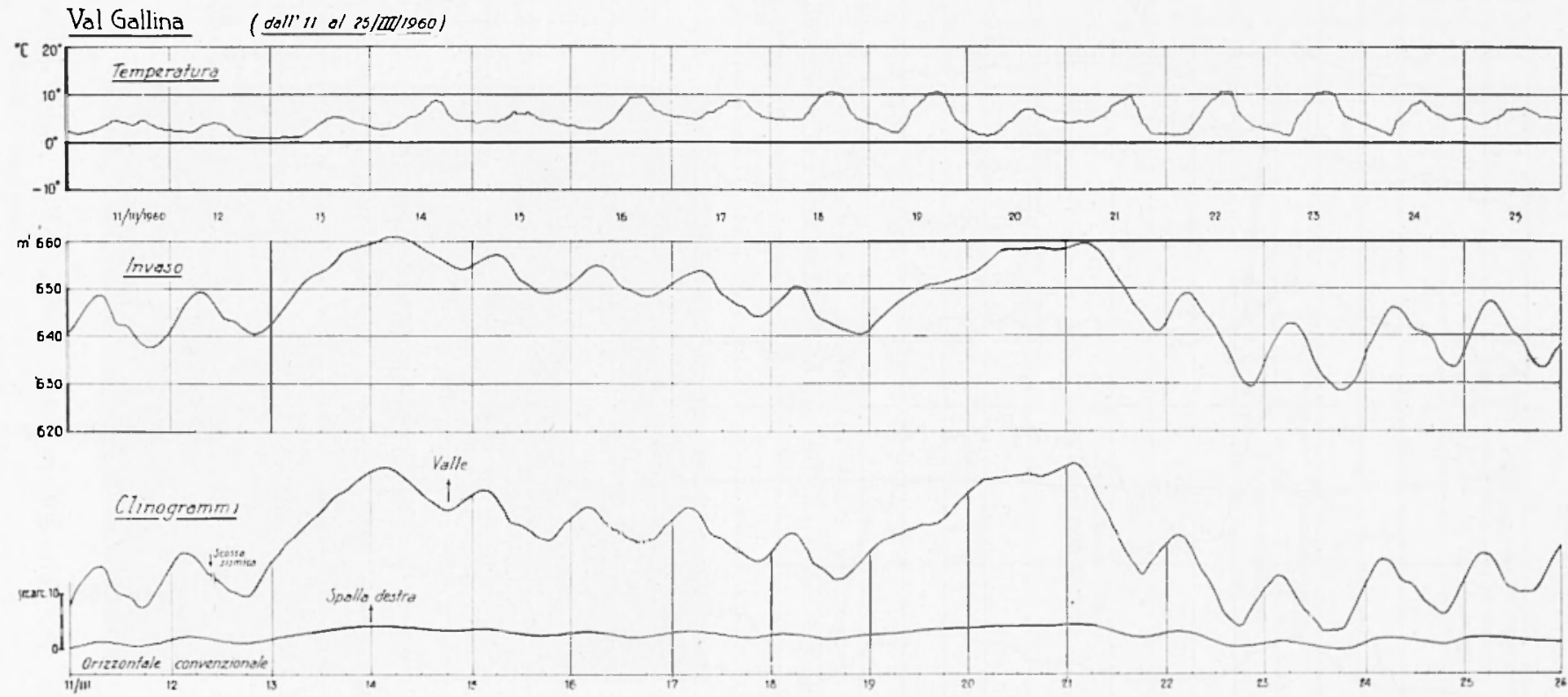

Fig. 26 - Diga di Val Gallina (affluente del Piave): la componente valle- monte del clinografo alla base del concio rentrale, registra fedelmente le variazioni del livello dellacqua nel hacino (invaso): ad mia spinta verso valle, corrisponde una lievissima flessione verso la spalla destra. 


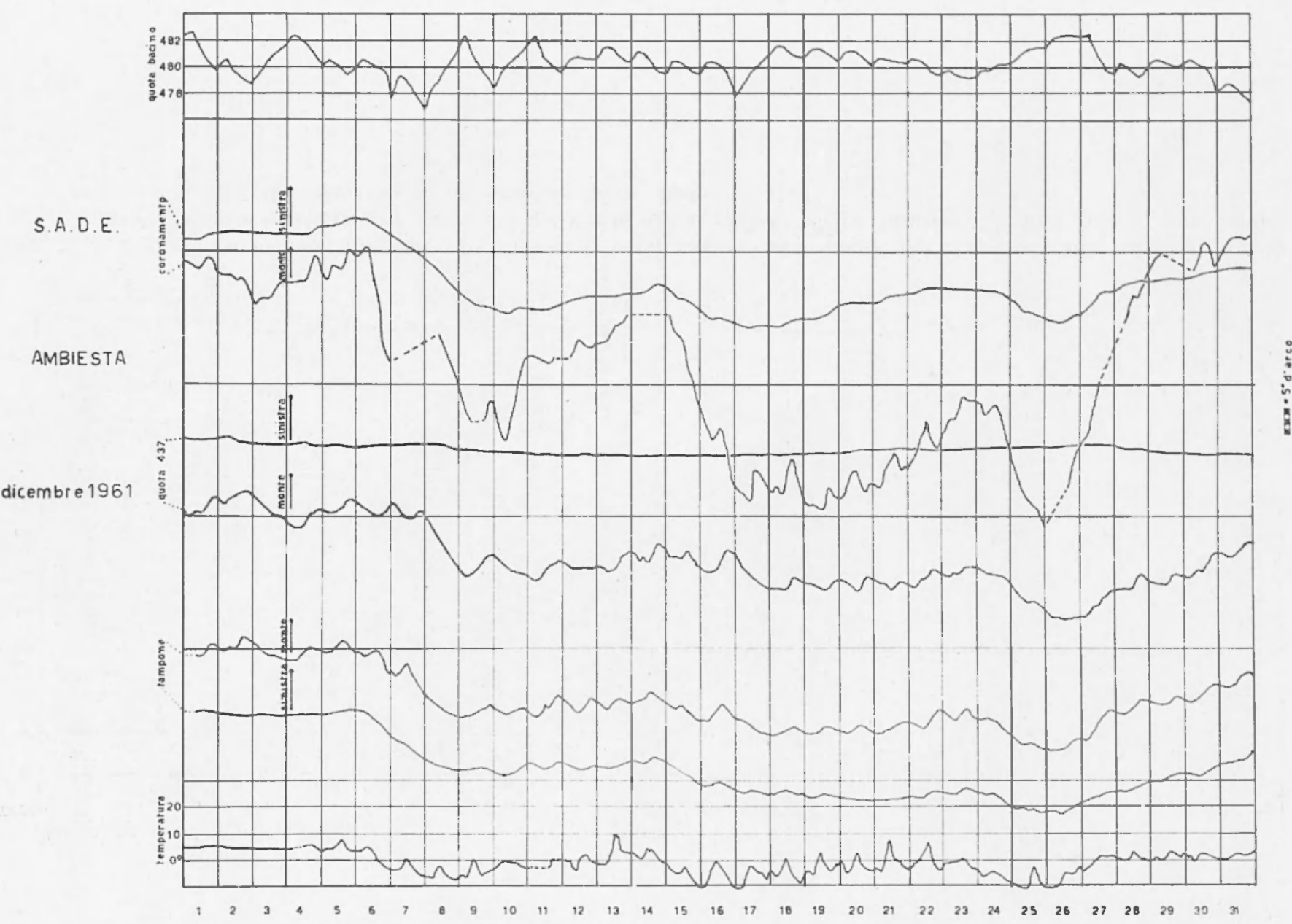

Nigr 27 - Diga doll Ambiesta (affluente del Tagliamento): in Dicembre (come in altre dighe) l"insolazione riesce inefficace (mancanza dellonda dimma). Oltre alle variazioni dell invaso (evidente dal coronamento al tampone, specie sulla componente valle-monte), si rende manifesta sulla diga anche la diminuzione della temperatura osterna, che si traduce in una contrazione del'a diga stessa, con flessione a valle. Resta provato il comportamento clel tampone. che segue anche in direzione spalla-spalla le variazioni di temperatura (v. testo). 


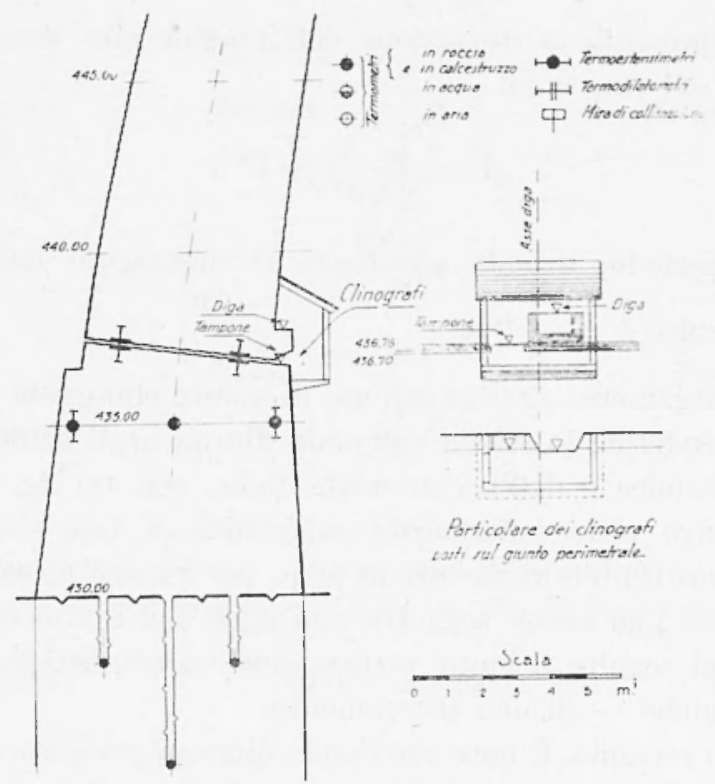

Fig. 28 - Diga dell'Ambiesta: sezione di parte del concio centrale, con postazione del clinografo-riga $(4 .+37,5)$ a del clinografo-tampone.

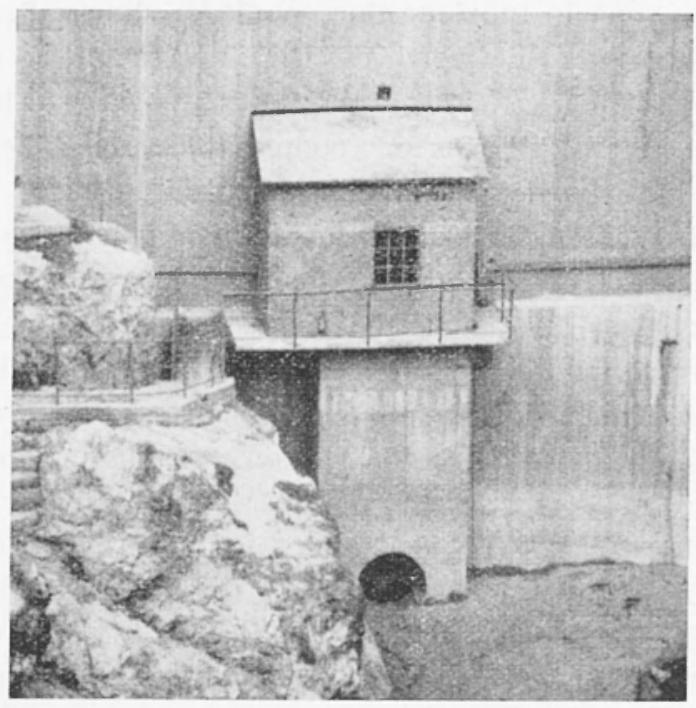

Fis: 28 bis - Diga dell'Ambiesta: casetta-custodia dei clinografi diga (1. 437.5$)$ a tampone. 
Se $\delta$ rappresenta la deviazione dell'magine alla distanza di registrazione di $100 \mathrm{~cm}$, si ha

$$
\delta=\pi 5 \frac{g}{\pi^{2} l} \psi T^{2}
$$

dove $T$ '̀ il periodo, avendo effettuato il montaggio del pendolo in modo che risulti $I=\frac{4}{9} l^{2} \cdot m$.

Ho già largamente riferito sull'uso di questo clinografo per la ricerca. dei blochi geodetici, lo studio dell'onda diuma negli edifici, dell'azione di flessione termica o dell:invaso nelle dighe, ecc. (v. fig. 26).

Qui ritengo utile soffermarmi sull'utilità di tale strumento, non solo come insostituibile rivelatore di ogni, per quanto piccola, variazione di flessione cui può essere soggetta una diga, ma anche quale prezioso coadiutore nel seguire il lento variare delle caratteristiche fisiche - e quindi dinamiche - di uno sbarramento.

Valga un esempio. È noto che l'onda diurna è presente con ampiezza crescente, dal tampone verso il coronamento della diga (dove scompare solo nei mesi di pieno inverno (fig. 27), quando l'insolazione è insufficiente per l'azione meccanica flettente). Il tampone, generalmente, ha un comportamento a sé $\left.{ }^{21}\right)$. Ciò riesce oltremodo netto nella diga dell'Ambiesta. In questa diga, sono in funzione due coppie di clinografi, alla distanza minore di 1 metro una sulla verticale dell'altra: senonché, mentre una è solidale con la base del concio centrale, l'altra poggia sul tampone (pulvino) (figg. 28 e 28 bis). La prima coppia (base del concio) pressoché non registra l'onda diurna. L'altra, un metro più in basso, registra chiaramente l'effetto dell'insolazione su entrambe le componenti. La spiegazione è la seguente. L'onda diurna sulla componente valle-monte è dovuta. all'allungamento per dilatazione del concio durante l'insolazione e alla sua flessione verso monte, il fenomeno contrario verificandosi ad insolazione ultimata. L'onda diurna sulla componente spalla-spalla è da attribuire all'asimmetria dell'insolazione stessa, che indugia molto a lungo sul lato sinistro della diga, anche quando il lato destro è già in ombra (v. figg. 29 e 30); asimmetria che resta tale anche nell'azione dinamica. Furono eseguite numerose risultanti del moto del tampone, soggetto agli effetti dell'insolazione. Gli esempi delle figg. 31-33 si riferiscono al 1957 a al 1958. Si noterà che le ellissi che ne conseguono hanno l'asse maggiore diretto nel primo (e terzo) quadrante e che la sua inclinazione rispetto alla linea monte-valle varia fra $52^{\circ}$ e $35^{\circ}$ circa. Col passare del tempo tale inclinazione è andata aumentando, mentre l'ellissi si faceva 
$4 \cdot 5 / 111 / 1957$

$15-16 / 1 \times / 1957$

$3-4 / x / 1957$
Digo
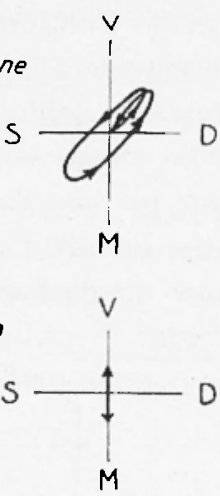

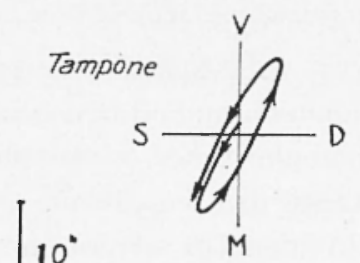

M

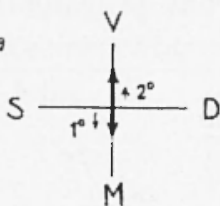

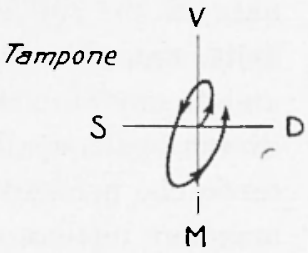

Digo

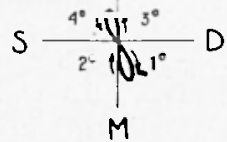

Fig. 31 - Diga dell'Ambiesta: composizione del movimento diumo (insolazione) a quota postazione diga (q. 437,5$)$ e tampone (q. 436,7).

$$
5-7 / \times / 1957
$$

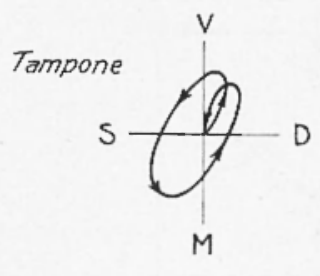

Diga

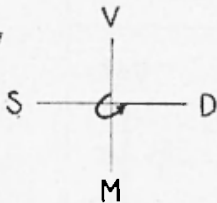

$$
10-11 / x / 1057
$$

$14-15 / \times 1 / 1957$

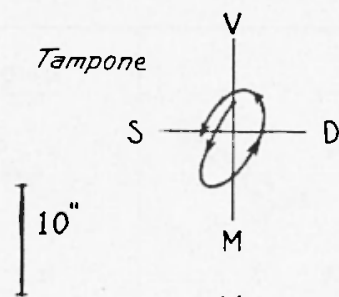

Digo

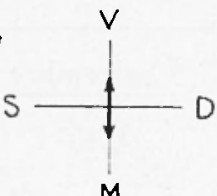

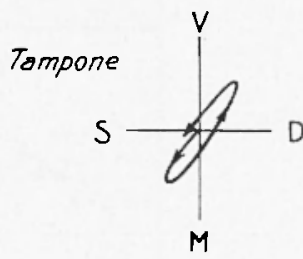

Diga

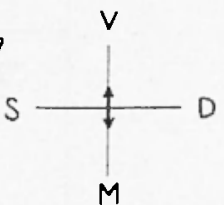

Fig. 32 - Diga dell Ambiesta: altri esempi di eomposizione dei movimenti diurni, in due postazioni a meno di un metro sulla stessa verticale.

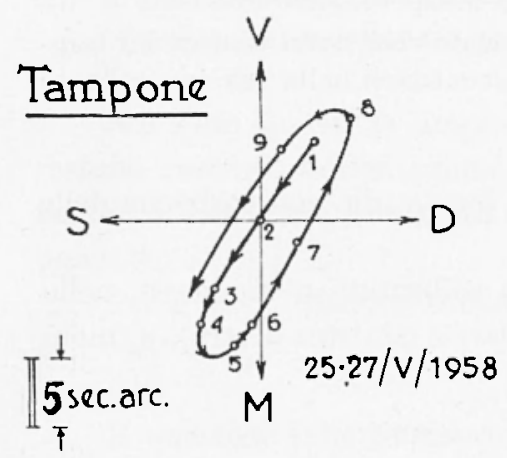

Fig. 33 - I)iga dell'Ambiesta: moto associato all'onda diurna sul tampone (1958). 
di più in più schiaceciata, per ridursi nel 1960 and un moto rettilineo inclinato di t5o sull'asse predetto (figge. 3.t, 35). Nel flattempo l'ampiezza della sinusoide onda diurna valle-monte, dapprima preponderante, è andata gradatanente diminuendo, lino ad uguagliare l'ampiezza dell'onda diuma spalla-spalla. Il fenomeno tende a proseguire nello stesso senso, tanto che nel 1961 la risultante torma a diventare ellittica, mal con l'asse maggiore inclinato più di $45^{\circ}$ rispetto all'asse monte-valle; mentre l'ampiezza dell'onda diurna è ora prevalente nella direzione spalla-spalla.

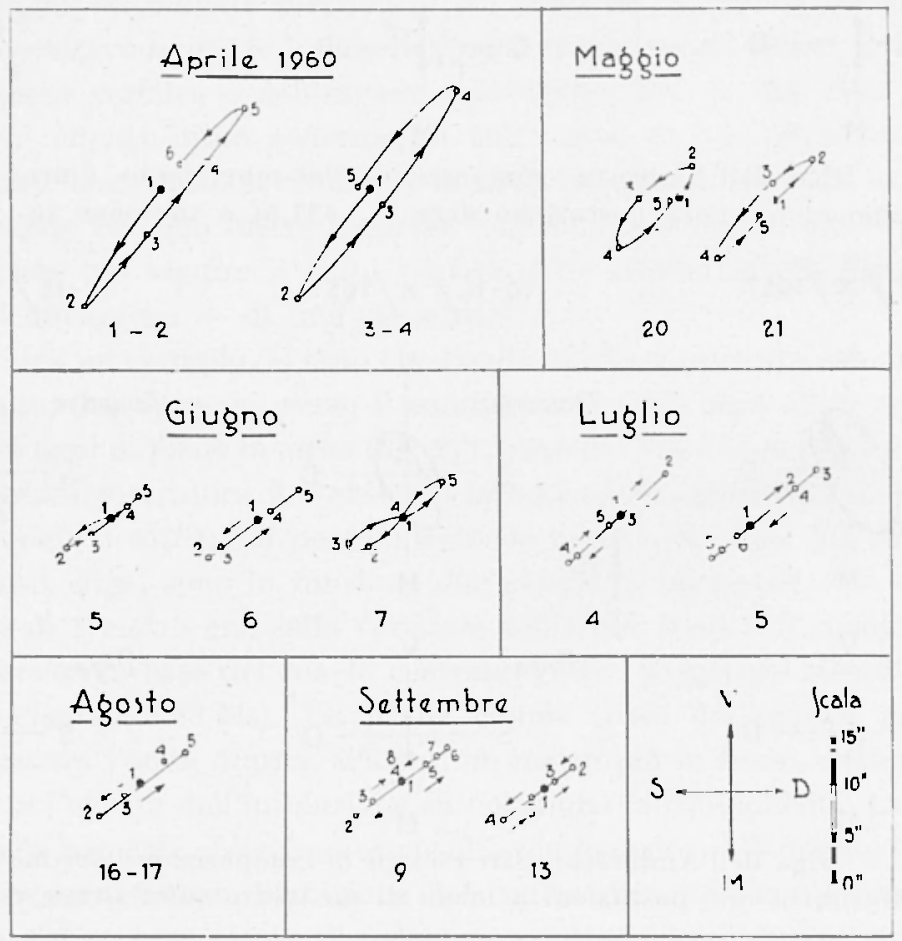

Fig. 34 - Diga dell Ambiesta: il moto provocato dallonda diurna sul tampone evolve nel tempo, tendendo ad aceentuarsi nella sua componente spalla-spallit.

L'interpretazione dei fatti osservati è legata alla composizione delle vibazioni armoniche rettangolari.

Supponiano che una particella $P$ sia sollecitata a muoversi, nello stesso tempo, lungo due direzioni rettangolari xi (sinistra-destra), y (monte-valle) seconclo le leggi di movinnento

$$
x=a \sin (a) t+\left(a_{1}\right) \quad, \quad y=b \sin \left((0) t+a_{2}\right) .
$$


Il punto $P$ rlescrive allora nel piano $x, y$ e nel tempo $T=\frac{2 \pi}{\omega}$, periodo comme ai due spostamenti $x, y$, una traiettoria chiusa di (xulazione

$$
\frac{x^{2}}{a^{2}}+\frac{y^{2}}{b^{2}}-\frac{2 x y}{a b} \cos \varphi \cdot-\sin ^{2} \varphi=0
$$

essendo $\varphi=\iota_{1}-a_{2}$.

La (*) rappresenta un'ellisse inseritta nel rettangolo definito dalle quattro rette $x= \pm a, y= \pm b$ (fig. 36 ).
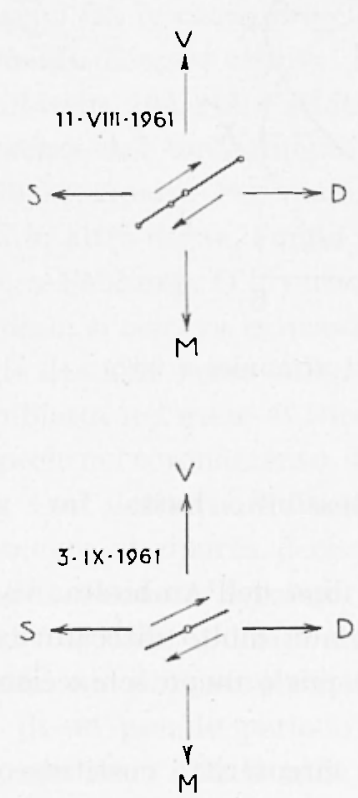
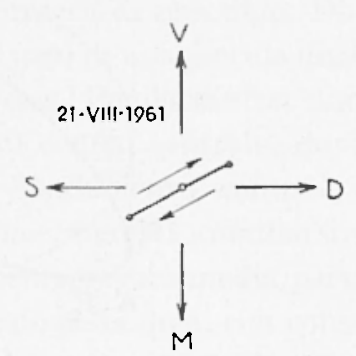

$5^{\prime \prime}$

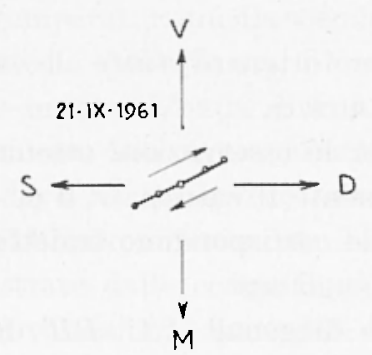

Fig. 35 - Diga dell Ambiesta: altre testimonianze sul progressivo prevalere della componente spalla destra-spalla sinistra sulla componente vallemonte, nel movinento provocato sul tampone dallonda diurna.

Nel caso in eni le ampiezze $a$ e $b$ (che noi supporremo positive) restano costunti, l'orientazione e la grandezza dell'ellisse traiettoria dipendono esclusivamente dalla differenza di fase $\varphi$ delle vibrazioni componenti.

$\operatorname{Per} \varphi=0$ l'ellisse si riduce alla diagonale $A A$, in quanto $\left(^{*}\right)$ diviene $\left(\frac{x}{a}-\frac{y}{b}\right)^{2}=0$.

Ii: questo il caso verificatosi nel 1960 . 
Per $\varphi=\frac{\pi}{2}$, la (*) diviene un'ellisse riferita agli assi coordinati ed $a$ a $b$ ne divengono i semiassi. Par $a=b$ l'ellisse si trasforma in eirconferenza.

Per $\varphi=\pi$ la (*) prende la forma $\left(\frac{x}{a}+\frac{y}{b}\right)^{2}=0$, e l'ellisse degenera nella diagonale $B B^{\prime}$ del rettangolo.

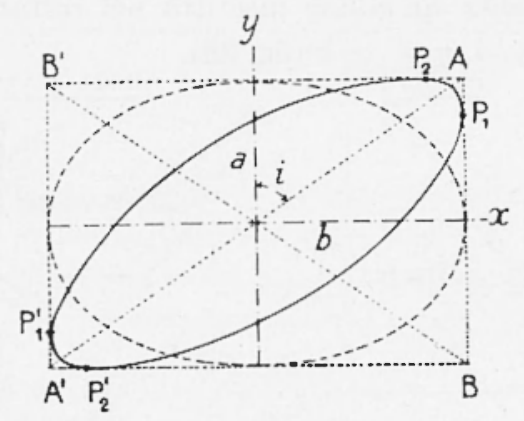

Fig. 36 - Composizione di moti armonici a $90^{\circ}$.

Per ottenere tutte le traiettorie possibili, basta far variare $\varphi$ da 0 a $\pi$.

Per le osservazioni ottenute presso la diga dell'Ambiesta, vale evidentemente il caso $p=0$, comunque, $\varphi$ non molto discosto da zero, al quale corrispondono traiettorie ellittiche più o meno schiacciate, non riferite agli assi.

Le diagonali $A A$ ', $B B^{\prime}$ del rettangolo circoscritto costituiscono un sistema di diametri coniugati per la famiglia di ellissi (*).

Nel caso in eni si verificano variazioni nell'ampiezza delle due sinusoidi, poiché $\frac{a}{b}=\operatorname{tang} i$, è chiaro che - per variazioni di fase pressoché nulle (come è il caso in esame)-per $a \leqq b$ è $i=45^{\circ}$, come appunto si è verificato dal 1957 al 1961.

Negli amni considerati, le ellissi traiettorie hamno subito variazioni sostanzialmente legate alle variazioni delle ampiezze dei moti componenti. E da chiedersi a cosa va attribuito il progressivo prevalere dell'ampiezza destra-sinistra su valle-monte. A prima vista sembra doversi licercare la causa in ma maggiore efficacia dell'insolazione (che agisce, come si è visto, in modo asimmetrico) sul calcestruzzo. 
Se però si osserva che, sostanzialmente, negli ultimi quattro anni la componente spalla-spalla ha conservato ampiezze pressoché costanti, mentre è stata la componente valle-monte che è andata progressivamente scemando di ampiezza, appare più giusto attribuire il fatto ad un progressivo irrigidimento del ealcestruzzo, cosi da giustificare la sua scemata attitudine a flettere sottio l'azione delle variazioni termiche, conseguenti all'insolazione. In un modo o nell'altro, i fatti constatati presuppongono una variazione nel tempo delle caratteristiche del calcestruzzo, tali da giustificure il fenomeno su detto.

Le variazioni della temperatura dell'aria e l'andamento dell'insolazione, sono fra le cause principali dei movimenti di una diga. Di esse ho ampiamente discusso altrove. Riporto qui il caso di una piccola diga, quella dell'Ambiesta, più volte eitata. Anche in essa, l'onda diuma diminuisce in ampiezza dal coronamento alla base del concio centrale, dove praticamente scompare. Per quanto concerne l'andamento anmuale, come si verifica in altre dighe, l'onda diurna si riduce, fino ad annullarsi, da Novembre a Febbraio. D'inverno, quando la temperatura media perviene ad un minimo, si osserva la massima contrazione della diga, con conseguente sensibile flessione verso valle. Si veda il chiaro esempio fornito dalla diga dell'Ambiesta nel mese di Dicembre 1961 (fig. 27). La flessione riesce marcata, specie nel coronamento, da quando la temperatuma media scende sotto lo zero. Con il ritorno della temperatura media dell'aria sopra lo zero, il coronamento si riporta decisamente verso monte. L'onda diuma, associata all'insolazione, è pressoché scomparsa: essa non figura infatti nella componente sinistra-destra del tampone, dove risulta invece (come si è visto) molto sviluppata durante i mesi da Febbraio a Novembre. Le oscillazioni di un pseudo periodo diurno, registrate dalle componenti vallemonte, non sono infatti onde diurne, bensì variazioni coperiodali dell'invaso. A questo riguardo, si noti come esse vengano trasmesse attenuate al tampone dalla base del concio (quota 437), su esso poggiante.

5. - Ho mostrato in lavori precedenti l'interesse di valutare, nei suoi riflessi geofisici, le misure geodetiche, che periodicamente vengono eseguite intorno alle grandi dighe. Cosi, le misure degli spostamenti orizzontali di punti determinati, quali risultano dalle triangolazioni, possono servire per il calcolo della deformazione orizzontale per ogni triangolo, nonché la grandezza e la direzione degli elementi camatteristici della deformazione elastica: dilatazione, rotazione, distorsione e deformazionc principale. Se $u$ e $v$ sono le componenti dello spostamento di un punto trigonometrico, con piferimento ad un arbitrario sistema di assi $x, y$, 
indicate con $A, \varepsilon, s$, ece. rispettivamente la dilatazione, la rotazione, la distorsione, ece., si ha ('s):

$$
A=\frac{\partial u}{\partial x}+\frac{\partial v}{\partial y} ; 2 \varepsilon=\frac{\partial u}{\partial y}-\frac{\partial v}{\partial x} ; 2 S=\frac{\partial v}{\partial x}+\frac{\partial u}{\partial y}:
$$

eec. ece.

Si può cosi determinare lentità delle tensioni che si genemono nella roccia in un intorno della diga, in corrispondenza degli spostamenti osservati; e valutare quando esse sono tali da condure olt re il lintite di rottura, sotto forma di piceole scosse sismiche.

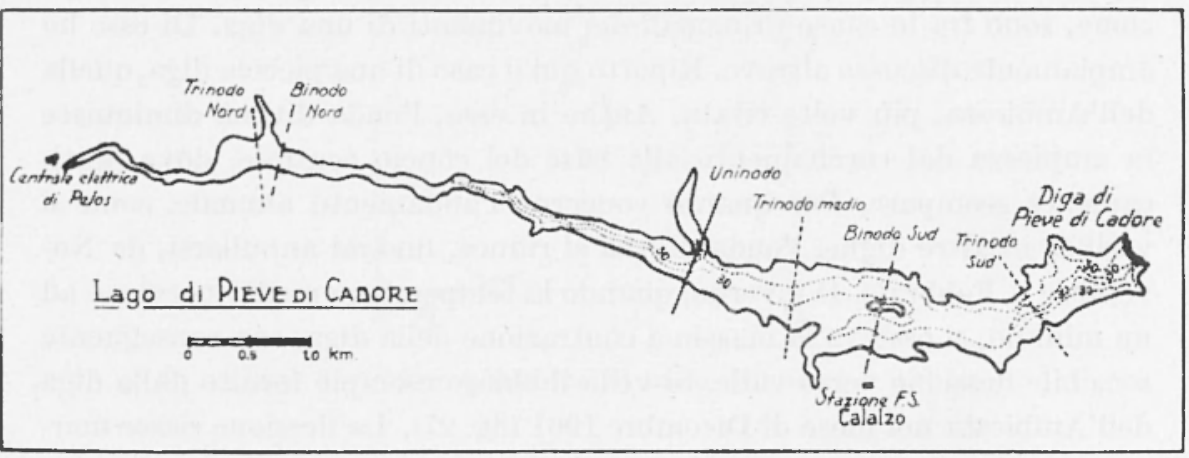

Fig. 37 - Lago di Pieve di cadore: posizione dei nodi delle sesse uni-, bie trinodali.

Un'ampia ricerea compinta sulle prime otto triangolazioni eseguite presso la diga di Pieve di Cadore, ha portato a notevoli risultati, aleuni dei quali del tutto imprevisti. Non mi soffermerò su di essi, discussi nel lavoro in eui sono riportati $\left({ }^{18}\right)$. Mi limitero ad osservare che - normalmente - ogni triangolazione è caratterizzata da un solo segno (positivo o negativo) pere la dilatazione. Ciò sta ad inclicare che, in tutta la zona sottoposta a misure geodetiche, intorno alla diga, si ha contemporaneamente o espansione del mezzo (dilatazione positiva) o contrazione. Il variare della misura o della direzione di tali fenomeni, dà hogo a distorsioni, pì̀ o meno accentuate. Gli assi principali delle ellissi di deformazione (tese o contratte) esprimono, con le loro lunghezze a direzioni, lentità della deformazione clastica e l'azimut secondo cui agisce.

6. - Si è già osservato che anche laidrodinamica può portare il suo valido contributo, per configurare compiutamente l'ambiente geofisico associato arl una grande diga. 
L'acequa contenuta in bacini chiusi o commene aperti (mali interni, langhi, golfi, batie, rade, ece.) può divenir sede di moti liberi, capaci di tenerla in movimento sul ritmo di periodi svariatissimi, legati alla forma dei bacini a alla profondità dell'acequa. La vasta letteratura sull'argomento considera un gran numero fina i casi che si possono presentare, alcuni dei

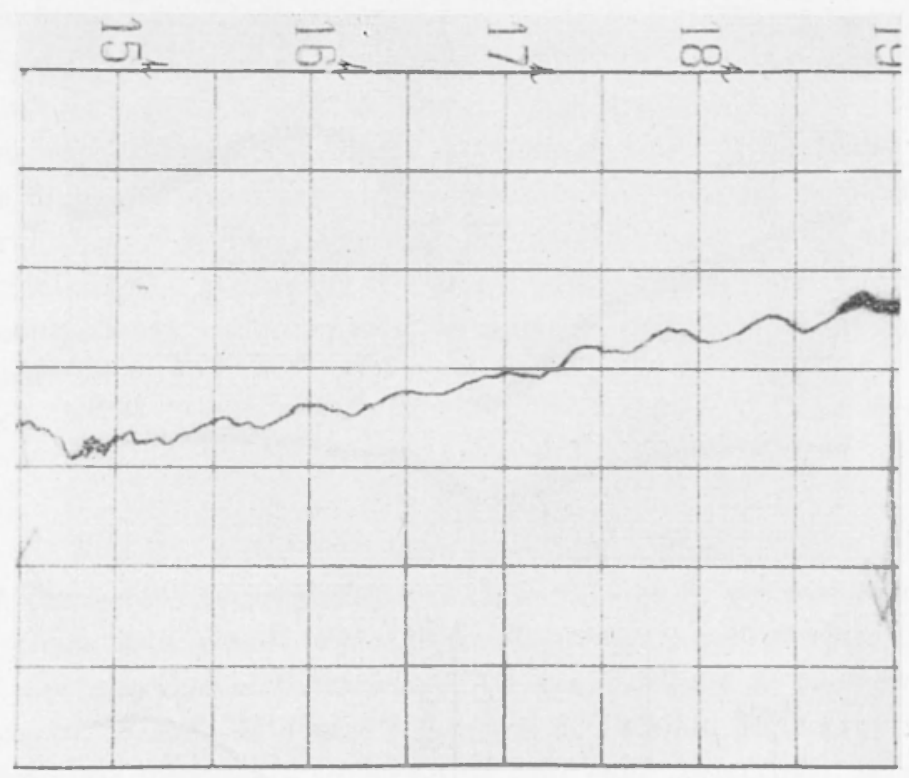

Fig. 38 - Lago di Pieve di cadore: esempio di lieve minodale dal lato della diga.

quali risultano alquanto complesssi. Nella teoria di Chrystal, l'equazione fondamentale che regge il moto stazionario in bacini chiusi i della forma

$$
\sigma(v) \frac{d^{2} u}{d v^{2}}+\frac{4 \pi^{2}}{g T^{2}} u=0
$$

dove $u=\xi \cdot S(x)$, essendo 5 esporessione degli spostamenti orizzontali delle particelle di una generiea sezione $s$ ' $(x), T$ is il periodo del moto libero, g l'accelerazione di gravita, $v=l b(x) d x$, essendo $b(x)$ la larghezza variabile del bacino, misurata alla superticie libela, e $\sigma(v)=S(x) \cdot b(x)$. La rurva rappresentata dalla funzione $\sigma(v)$ i detta la curere nomale. 
La determinazione delle oscillazioni libere nei bacini chiusi è quindi ricondotta alla risoluzione di un'equazione differenziale omogenea del second'ordine, con certe condizioni agli estremi. Esistono altri metodi, sui quali ritengo inutile di insistere in questa sede. Accennerò solo a

a)

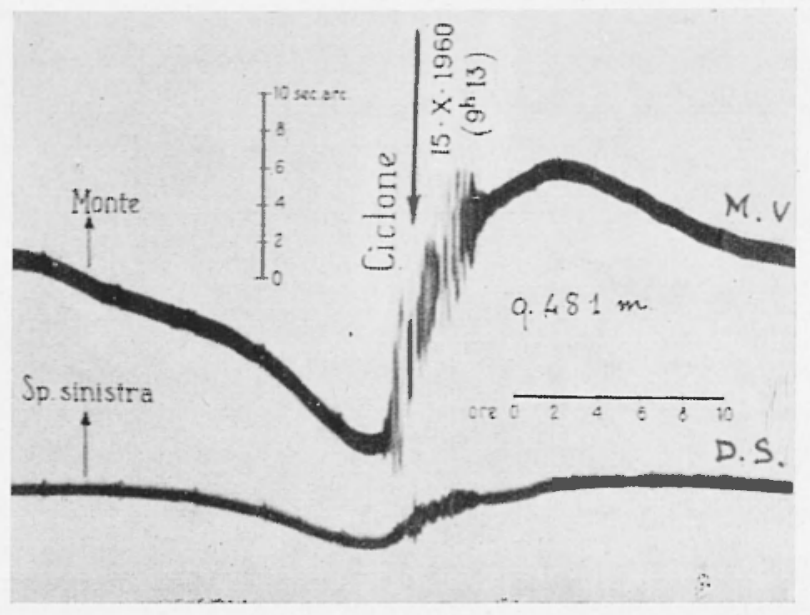

b)

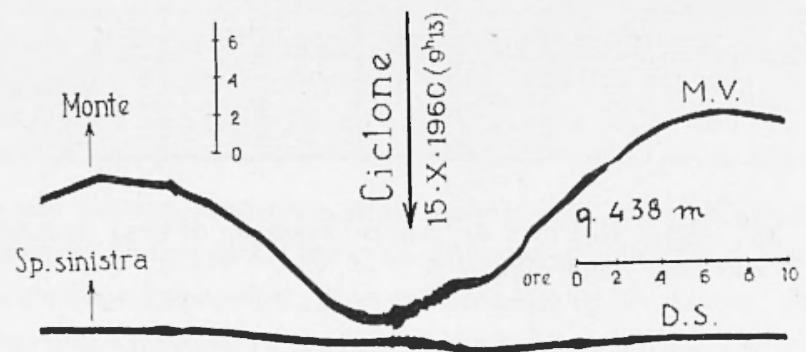

Fig. 39 - Diga dell'Ambiesta: l'azione del vento ciclonico (nel caso specifico, da valle) sulla diga e sul lago può determinare variazioni rapidissime della verticale apparente (vibrazioni), decrescenti dal coronamento $(a)$ al tampone $(b)$ e particolarmente ampie sulla componente valle-monte.

quelle delle inpedenze idrodinamiche di Neumann che, assieme a quello di Hidaka, fu applicato al lago Cadore $\left({ }^{14}\right)$, creato dallo sbarramento del Piave, compiuto nella stretta di Sottocastello (fig. 37). Le oscillazioni uninorali di detto lago furono dapprima registrate dai clinogmafi funzionanti nei conci della diga, sotto forma di variazioni della verticale apparente, aventi un periodo di mezz’ora cirea (`). I periodi delle oscillazioni 
libere uninodali e binodali, quali risultano dall'applieazione delle teorie idrodinamiche sulle sesse, sono

$$
\begin{array}{ll}
\text { oscillazione uninodale } & \text { periodo } 26,2 \mathrm{~min} . \\
\text { oscillazione binodale } & \text { periodo } 8,1 \mathrm{~min} .
\end{array}
$$

L'osservazione compinta dal lato della diga (dove le sesse presentano minore ampiezza) mediante apposito limnografo, ha portato, per il periodo dell'uninodale, ad un valore di $28-30 \mathrm{~m}$ circa (fig. 38). Saranno realizzate, a questo proposito, osservazioni sistematiche.

Va infine notato che il lago in burasca può - specie nel caso di piccole dighe - provocare rapide flessioni, chiaramente registrabili dai clinografi. Presso la diga dell'Ambiesta, non mancano esempi di tali perturbazioni, che - per evidenti motivi - sono particolarmente sensibili sulla componente valle-monte e scemano d'ampiezza dal coronamento alla base della diga (fig. 39).

\section{RIASSUNTO}

Il sismografo (vibrografo) e il clinografo quali preziosi coadiuvanti nal problema delle grandi dighe. Come il vibrografo può rivelare i periodi propri dominanti nelle stratificazioni rocciose, destinate ad ancorare grandi sbarramenti. Modulo di Young e rapporto di Poisson, dedotti con l'uso del vibrografo, che consente la registrazione delle onde trasversali, permettendo il raggiungimento di una maggiore approssimazione, nella determinazione delle costanti elastiche del mezzo solido. Decadimento del modulo elastico della roccia, in seguito ai lavori di sbancamonto e al comportamento della diga sotto l'azione delle flessioni provenienti dall'invaso e dall'andamento termico stagionale e diurno.

Il problema della trasmissione dell'energia elastica dalla roccia all'acqua e viceversa: esperienze eseguite sul lago di Sauris (Lumiei). Appartenenza della roccia d'imposta ad un solo blocco geodetico: come il clinografo può controllare questa opportuna richiesta. Utilità di una stazione sismica funzionante presso un grande sbarramento; sua utilizzazione ai fini di un costante, sensibilissimo controllo, e nella valutazione dell'energia associata alle minutissime fratture provocate nella roccia dal giuoco alterno delle flessioni della diga, rivelate dalle stazioni clinografiche funzionanti nei suoi cunicoli e sulle sue spalle. Casi delle dighe di Pieve di Cadore e del Vajont. Funzione delle stazioni clinografiche e sismografiche dopo la costruzione della diga: controllo continuo delle condizioni di equilibrio ambientali. 
Dighe di Pieve di Cadore, I 'al Gallina, Ambiesta, Pontesei, ece. Interpretazioni geofisiche di misure geodetiche, con particolare riguardo alla determinazione degli elementi della deformazione elastica: dilatazione, rotazione, distorsione ed assi principali della deformazione. Eventuale variazione nel tempo delle caratteristiche elastiche di una diga: loro controllo mediante il periodico rilevamento dei periodi delle oscillaziomi libere trasversali dei conci della diga. - Lidrodimamica al servizio delle grandi dighe: rilevamento delle caratteristiche mincipali delle oscillazioni libere (sesse) del bacino idrico, consegnente alla costruzione di un grande sbaramento. Applicazione al lago Cadore, formato dallo sbarramento della valle del Piave, a Pieve di Cadore.

\section{SU.WMAKY}

The seismograph (vibrograph) and the rlinograph as arecions helps in the problem of large dams. - How a vibrograph mey reseal the dominant periods of the rochy layers, which shall aiechor large barrages. - Modulus of Young and Poisson ratio, obtained though the ribrograph, which allows

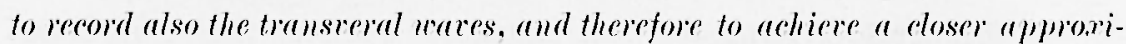
mation in the determination of elastic characteristics of the solid medium. Deray of the elastic modulus of the moli, due to brealing worls and to the beharioue of the dem under the action of the flexions from the hydrie basin and the seasomal and daily thermire cycle. - Pertainanee of the rock to a single geodetic block: how the clinograph may chect this oppontune request. How the elastic energ!y is captured from the hydrie basin and forrarded: errpenienees from the lale of sauris. - Ctility of a norling seismic station by a large dam: its application with regerd to a constant, very sensilive contres? and to the exaluation of the energ! connected to the besy small fractures caused in the rock by the alternate Aerions of the dam, revealed by the clinograph stations functioning in its galleries and behind it. The dams at Pieve di Cadore and the Vajont.-- Function of clinographic and seismographic stations after the building of the dem: continous control of the cquilibrium of the area. Dams at Pieve di Cadore, Val Gallima, Ambiesta, Pontessei, rte. - Geophysical interpretations of grodetical measurements, with special regard to the determination of the elements of the elastic deformation: dilutation, rot:ation, shear and main axes of the deformation. -- Possible peentual raviation of a dam's elastic characteristics: control through periodical survey of the periods of free, transwersal oscillations of the dam's section. - Hydrodinamies for large dams: survey of the main features of free vibrations (seiches) 
of the hydric basin, consequent to the construction of " large barage. Application to the latie Catore, formed by the barrage of the Piare calley, at Pieve di Cardore.

\section{BIBLIOGRAFIA}

(1) CAlo1 P.. Il pendolo orizzontale eome clinometro. "Annali di Geofisican", III, $(1950)$.

(2) Calor P., Deferminazione del Jodulo di Foung eol melodo sismico. "Rolazioni e Studi ", dell'Vfficio Studi SADE. 11. I, (Dicombre 1951).

${ }^{3}$ ) Caroi P., Ricerehe sismologiche presso la diga di Pieve di Cadore. "Rolaz. o sturli ", n. 9, (1952).

(1) Ca cor P., Riecrehe clinografiche presso le diga di Pieve di Cadore. "Rolaz. a Stucli ", 11, 12, (1952).

$\left.{ }^{5}\right)$ Canor P', Fibrazioni della diga del Lamiei durame lapertura dello searieo di fondo. "Relaz. o Studli ", n. 6. (1953).

(6) Canor P., Esperienze nella stretta del Wis. "Relaz. e Studi", n. 10, (1953).

(`) Canor P., Osservazioni sismiche e elinografiche presso grandi dighe di sbarramento. "Annali di Geofisican, VI, 3, (1953).

$\left.{ }^{8}\right)$ Casor P., Esperienze geosismiche sul ghiacciaio della Mrtrmolada. "Rolaz. e. Stucli ", 11. 19, (1954).

(9) (Anor P., Esperienze geosismiche nella walle del Cordevole (Caprile), "Relaz. " Sturli", 11. 21, (1954).

(10) Calor P'., Spadea M. C., Decadimento del modulo elastico in roceia a con. latto con bacint idrici artifiriali. "Ammali ali Geofisican. VI, 4, (1954).

(11) Canol P., Spadea .I. C., Relazioni fra lente varazioni dinclinazione e moti sismici in zona ad elevalu sismicila. "Rentl. Ace. Naz. dei Lincei ", XVIII. 3, (I955).

(12) Caron P., Sismicitì della zona di Tolmezzo. "Relaz. O Studi SADE", 11. 7. (Nuova serie), (1955).

('3) CaLor P., Il eomportamento delle grandi dighe dal punto di vista geofisioo. " J'Aequa ". 1-2, (1955).

(1.) Pezzori G. A., Oscillazioni libere del lago di Piece di Cadore. "Archivio th Oceanografia e Limmologia ", X. 1-2, (1955).

(15) CAIor P., Il jotoclinograjo a pendolo orizzontale nella teoria e nell applicazione. "Jinergia Elettrica ", XXXIII, 10, (1956).

(16) Catol P.. Sui periodi doscillazione libera dei conci di una diga e sulle loro relazioni con le caratteristiche elastiche del ealeestruzzo. "L'Energia Elettrica ", XXXIII, 12. (1956).

$\left.{ }^{(17}\right)$ Canor P'. Sulla dispersione delle onde sismiche nell'ambito delle allissime trequenze. "Annali di Geofisica ", X. 3-4, (1957). 
(18) Caloi P., Spadea M. C., Geophysical Interpretations of the first Series of Triangulations carried out at the Pieve di Cadore Dam. "VI Congrès des Grands Barragres ", New York, 1958.

(19) Calor P., Come la geofisica può contribuire ai problemi concernenti la costruzione e l'osservazione delle grandi dighe. "Relazioni e Studi ", n. 19, (Nuova Serie), (1958).

${ }^{(20)}$ Calor P., About some phenomena preceding and following the seismic movements in the zone characterized by high seismicity. "Contributions in Geophysics: In Honor of Beno Gutenhergn. I'ergamon Press, Londra, 1958.

(21) Caloi P'., Osservazioni clinografiche presso la diga dell'Ambiesta. "Relazioni e Studi ", 1. 21, (Nuova serie), (1959).

(22) Calor P., La geofisica e le grandi dighe. "I'Energia Elettrica", XXXIX, $1,(1962)$.

(23) Kana K., Tanaka T. and Yosnizawas., Comparalive Studies of Earthqualie Motions on the Ground and Underground. "Bull. Earthq. Res. Inst. ", 37, (1959).

(24) SIImozuru D., Elasticity of Rocks under Initial Stresses, with Special Reference to the Fracture Problem. "Bull Earthq. Res. Inst. ", XXXIII, 3 , (1955). 